\title{
Search for ultrahigh-energy tau neutrinos with IceCube
}

R. Abbasi, ${ }^{28}$ Y. Abdou, ${ }^{23}$ T. Abu-Zayyad,${ }^{34}$ M. Ackermann, ${ }^{42}$ J. Adams, ${ }^{16}$ J. A. Aguilar, ${ }^{22}$ M. Ahlers, ${ }^{28}$ D. Altmann, ${ }^{1}$ K. Andeen, ${ }^{28}$ J. Auffenberg ${ }^{28}$ X. Bai, ${ }^{32, *}$ M. Baker, ${ }^{28}$ S. W. Barwick,${ }^{24}$ V. Baum,${ }^{29}$ R. Bay, ${ }^{7}$ K. Beattie,${ }^{8}$ J. J. Beatty,${ }^{18,19}$ S. Bechet, ${ }^{13}$ J. K. Becker, ${ }^{10}$ K.-H. Becker, ${ }^{41}$ M. Bell, ${ }^{39}$ M. L. Benabderrahmane, ${ }^{42}$ S. BenZvi, ${ }^{28}$ J. Berdermann, ${ }^{42}$ P. Berghaus, ${ }^{32}$ D. Berley, ${ }^{17}$ E. Bernardini, ${ }^{42}$ D. Bertrand, ${ }^{13}$ D. Z. Besson,${ }^{26}$ D. Bindig, ${ }^{41}$ M. Bissok, ${ }^{1}$ E. Blaufuss, ${ }^{17}$ J. Blumenthal, ${ }^{1}$ D. J. Boersma, ${ }^{1}$ C. Bohm,${ }^{35}$ D. Bose,${ }^{14}$ S. Böser,${ }^{11}$ O. Botner, ${ }^{40}$ L. Brayeur, ${ }^{14}$ A. M. Brown, ${ }^{16}$ S. Buitink, ${ }^{14}$ K. S. Caballero-Mora, ${ }^{39}$ M. Carson, ${ }^{23}$ M. Casier, ${ }^{14}$ D. Chirkin, ${ }^{28}$ B. Christy, ${ }^{17}$ F. Clevermann, ${ }^{20}$ S. Cohen, ${ }^{25}$ D. F. Cowen, ${ }^{39,38}$ A. H. Cruz Silva, ${ }^{42}$ M. V. D'Agostino, ${ }^{7}$ M. Danninger, ${ }^{35}$ J. Daughhetee, ${ }^{5}$ J. C. Davis, ${ }^{18}$ C. De Clercq, ${ }^{14}$ T. Degner, ${ }^{11}$ F. Descamps, ${ }^{23}$ P. Desiati, ${ }^{28}$ G. de Vries-Uiterweerd, ${ }^{23}$ T. DeYoung, ${ }^{39}$ J. C. Díaz-Vélez, ${ }^{28}$ J. Dreyer, ${ }^{10}$ J.P. Dumm, ${ }^{28}$ M. Dunkman, ${ }^{39}$ J. Eisch, ${ }^{28}$ R. W. Ellsworth, ${ }^{17}$ O. Engdegård, ${ }^{40}$ S. Euler, ${ }^{1}$ P. A. Evenson, ${ }^{32}$ O. Fadiran, ${ }^{28}$ A. R. Fazely, ${ }^{6}$ A. Fedynitch, ${ }^{10}$ J. Feintzeig, ${ }^{28}$ T. Feusels, ${ }^{23}$ K. Filimonov, ${ }^{7}$ C. Finley,${ }^{35}$ T. Fischer-Wasels, ${ }^{41}$ S. Flis, ${ }^{35}$ A. Franckowiak, ${ }^{11}$ R. Franke, ${ }^{42}$ T. K. Gaisser, ${ }^{32}$ J. Gallagher, ${ }^{27}$ L. Gerhardt, ${ }^{8,7}$ L. Gladstone, ${ }^{28}$ T. Glüsenkamp, ${ }^{42}$ A. Goldschmidt, ${ }^{8}$ J. A. Goodman, ${ }^{17}$ D. Góra, ${ }^{42}$ D. Grant, ${ }^{21}$ A. Groß,${ }^{31}$ S. Grullon, ${ }^{28}$ M. Gurtner, ${ }^{41}$ C. Ha,${ }^{8,7}$ A. Haj Ismail, ${ }^{23}$ A. Hallgren, ${ }^{40}$ F. Halzen,${ }^{28}$ K. Hanson, ${ }^{13}$ D. Heereman, ${ }^{13}$ P. Heimann, ${ }^{1}$ D. Heinen, ${ }^{1}$ K. Helbing, ${ }^{41}$ R. Hellauer,${ }^{17}$ S. Hickford, ${ }^{16}$ G. C. Hill, ${ }^{2}$ K. D. Hoffman, ${ }^{17}$ B. Hoffmann, ${ }^{1}$ A. Homeier, ${ }^{11}$ K. Hoshina, ${ }^{28}$ W. Huelsnitz, ${ }^{17},{ }^{\dagger}$ P. O. Hulth, ${ }^{35}$ K. Hultqvist,${ }^{35}$ S. Hussain, ${ }^{32}$ A. Ishihara, ${ }^{15}$ E. Jacobi, ${ }^{42}$ J. Jacobsen, ${ }^{28}$ G. S. Japaridze, ${ }^{4}$ H. Johansson, ${ }^{35}$ A. Kappes, ${ }^{9}$ T. Karg, ${ }^{41}$ A. Karle, ${ }^{28}$ J. Kiryluk, ${ }^{36}$ F. Kislat, ${ }^{42}$ S. R. Klein, ${ }^{8,7}$ J.-H. Köhne, ${ }^{20}$ G. Kohnen, ${ }^{30}$ H. Kolanoski, ${ }^{9}$ L. Köpke, ${ }^{29}$ S. Kopper, ${ }^{41}$ D. J. Koskinen, ${ }^{39}$ M. Kowalski, ${ }^{11}$ M. Krasberg, ${ }^{28}$ G. Kroll ${ }^{29}$ J. Kunnen, ${ }^{14}$ N. Kurahashi, ${ }^{28}$ T. Kuwabara ${ }^{32}$ M. Labare,${ }^{14}$ K. Laihem, ${ }^{1}$ H. Landsman, ${ }^{28}$ M. J. Larson, ${ }^{39}$ R. Lauer, ${ }^{42}$ J. Lünemann, ${ }^{29}$ J. Madsen, ${ }^{34}$ R. Maruyama, ${ }^{28}$ K. Mase, ${ }^{15}$ H. S. Matis, ${ }^{8}$ K. Meagher, ${ }^{17}$ M. Merck, ${ }^{28}$ P. Mészáros, ${ }^{38,39}$ T. Meures, ${ }^{13}$ S. Miarecki, ${ }^{8,7}$ E. Middell, ${ }^{42}$ N. Milke, ${ }^{20}$ J. Miller, ${ }^{14}$ T. Montaruli, ${ }^{22,}$ R. Morse, ${ }^{28}$ S. M. Movit,${ }^{38}$ R. Nahnhauer, ${ }^{42}$ J. W. Nam, ${ }^{24}$ U. Naumann, ${ }^{41}$ S. C. Nowicki, ${ }^{21}$ D. R. Nygren,${ }^{8}$ S. Odrowski, ${ }^{31}$ A. Olivas, ${ }^{17}$ M. Olivo, ${ }^{10}$ A. O’Murchadha, ${ }^{28}$ S. Panknin, ${ }^{11}$ L. Paul, ${ }^{1}$ C. Pérez de los Heros, ${ }^{40}$ D. Pieloth, ${ }^{20}$ J. Posselt, ${ }^{41}$ P. B. Price, ${ }^{7}$ G. T. Przybylski, ${ }^{8}$ K. Rawlins,${ }^{3}$ P. Redl,${ }^{17}$ E. Resconi, ${ }^{31}$ W. Rhode,${ }^{20}$ M. Ribordy, ${ }^{25}$ M. Richman,${ }^{17}$ B. Riedel, ${ }^{28}$ J. P. Rodrigues, ${ }^{28}$ F. Rothmaier, ${ }^{29}$ C. Rott, ${ }^{18}$ T. Ruhe, ${ }^{20}$ D. Rutledge, ${ }^{39}$ B. Ruzybayev, ${ }^{32}$ D. Ryckbosch,${ }^{23}$ H.-G. Sander ${ }^{29}$ M. Santander, ${ }^{28}$ S. Sarkar, ${ }^{33}$ K. Schatto, ${ }^{29}$ M. Scheel, ${ }^{1}$ T. Schmidt, ${ }^{17}$ S. Schöneberg, ${ }^{10}$ A. Schönwald, ${ }^{42}$ A. Schukraft, ${ }^{1}$ L. Schulte, ${ }^{11}$ A. Schultes,${ }^{41}$ O. Schulz,${ }^{31}$ M. Schunck, ${ }^{1}$ D. Seckel, ${ }^{32}$ B. Semburg, ${ }^{41}$ S. H. Seo,${ }^{35}{ }^{\S}$ Y. Sestayo, ${ }^{31}$ S. Seunarine, ${ }^{12}$ A. Silvestri, ${ }^{24}$ M. W. E. Smith,${ }^{39}$ G. M. Spiczak, ${ }^{34}$ C. Spiering, ${ }^{42}$ M. Stamatikos, ${ }^{18, \|}$ T. Stanev, ${ }^{32}$ T. Stezelberger ${ }^{8}$ R. G. Stokstad, ${ }^{8}$ A. Stöß1,${ }^{42}$ E. A. Strahler, ${ }^{14}$ R. Ström, ${ }^{40}$ M. Stüer, ${ }^{11}$ G. W. Sullivan, ${ }^{17}$ H. Taavola,${ }^{40}$ I. Taboada, ${ }^{5}$ A. Tamburro, ${ }^{32}$ S. Ter-Antonyan, ${ }^{6}$ S. Tilav, ${ }^{32}$ P. A. Toale, ${ }^{37}$

S. Toscano, ${ }^{28}$ N. van Eijndhoven, ${ }^{14}$ A. Van Overloop, ${ }^{23}$ J. van Santen, ${ }^{28}$ M. Vehring, ${ }^{1}$ M. Voge, ${ }^{11}$ C. Walck,${ }^{35}$

T. Waldenmaier, ${ }^{9}$ M. Wallraff, ${ }^{1}$ M. Walter, ${ }^{42}$ R. Wasserman, ${ }^{39}$ Ch. Weaver, ${ }^{28}$ C. Wendt, ${ }^{28}$ S. Westerhoff,${ }^{28}$ N. Whitehorn, ${ }^{28}$

K. Wiebe, ${ }^{29}$ C. H. Wiebusch, ${ }^{1}$ D. R. Williams,${ }^{37}$ R. Wischnewski, ${ }^{42}$ H. Wissing,${ }^{17}$ M. Wolf,${ }^{35}$ T. R. Wood, ${ }^{21}$

K. Woschnagg, ${ }^{7}$ C. Xu, ${ }^{32}$ D. L. Xu, ${ }^{37}$ X. W. Xu, ${ }^{6}$ J.P. Yanez,${ }^{42}$ G. Yodh,${ }^{24}$ S. Yoshida, ${ }^{15}$ P. Zarzhitsky, ${ }^{37}$ and M. Zoll ${ }^{35}$

(IceCube Collaboration)

\footnotetext{
${ }^{1}$ III. Physikalisches Institut, RWTH Aachen University, D-52056 Aachen, Germany

${ }^{2}$ School of Chemistry \& Physics, University of Adelaide, Adelaide SA, 5005 Australia

${ }^{3}$ Department of Physics and Astronomy, University of Alaska Anchorage, 3211 Providence Dr., Anchorage, Alaska 99508, USA ${ }^{4}$ CTSPS, Clark-Atlanta University, Atlanta, Georgia 30314, USA

${ }^{5}$ School of Physics and Center for Relativistic Astrophysics, Georgia Institute of Technology, Atlanta, Georgia 30332, USA

${ }^{6}$ Department of Physics, Southern University, Baton Rouge, Louisiana 70813, USA

${ }^{7}$ Department of Physics, University of California, Berkeley, California 94720, USA

${ }^{8}$ Lawrence Berkeley National Laboratory, Berkeley, California 94720, USA

${ }^{9}$ Institut für Physik, Humboldt-Universität zu Berlin, D-12489 Berlin, Germany

${ }^{10}$ Fakultät für Physik \& Astronomie, Ruhr-Universität Bochum, D-44780 Bochum, Germany

${ }^{11}$ Physikalisches Institut, Universität Bonn, Nussallee 12, D-53115 Bonn, Germany

${ }^{12}$ Department of Physics, University of the West Indies, Cave Hill Campus, Bridgetown BB11000, Barbados

${ }^{13}$ Université Libre de Bruxelles, Science Faculty CP230, B-1050 Brussels, Belgium

${ }^{14}$ Vrije Universiteit Brussel, Dienst ELEM, B-1050 Brussels, Belgium

${ }^{15}$ Department of Physics, Chiba University, Chiba 263-8522, Japan

${ }^{16}$ Department of Physics and Astronomy, University of Canterbury, Private Bag 4800, Christchurch, New Zealand

${ }^{17}$ Department of Physics, University of Maryland, College Park, Maryland 20742, USA
} 
${ }^{18}$ Department of Physics and Center for Cosmology and Astro-Particle Physics, Ohio State University, Columbus, Ohio 43210, USA

${ }^{19}$ Department of Astronomy, Ohio State University, Columbus, Ohio 43210, USA

${ }^{20}$ Department of Physics, TU Dortmund University, D-44221 Dortmund, Germany

${ }^{21}$ Department of Physics, University of Alberta, Edmonton, Alberta, Canada T6G $2 G 7$

${ }^{22}$ Département de physique nucléaire et corpusculaire, Université de Genève, CH-1211 Genève, Switzerland

${ }^{23}$ Department of Physics and Astronomy, University of Gent, B-9000 Gent, Belgium

${ }^{24}$ Department of Physics and Astronomy, University of California, Irvine, California 92697, USA

${ }^{25}$ Laboratory for High Energy Physics, École Polytechnique Fédérale, CH-1015 Lausanne, Switzerland

${ }^{26}$ Department of Physics and Astronomy, University of Kansas, Lawrence, Kansas 66045, USA

${ }^{27}$ Department of Astronomy, University of Wisconsin, Madison, Wisconsin 53706, USA

${ }^{28}$ Department of Physics, University of Wisconsin, Madison, Wisconsin 53706, USA

${ }^{29}$ Institute of Physics, University of Mainz, Staudinger Weg 7, D-55099 Mainz, Germany

${ }^{30}$ Université de Mons, 7000 Mons, Belgium

${ }^{31}$ T.U. Munich, D-85748 Garching, Germany

${ }^{32}$ Bartol Research Institute and Department of Physics and Astronomy, University of Delaware, Newark, Delaware 19716, USA

${ }^{33}$ Department of Physics, University of Oxford, 1 Keble Road, Oxford OX1 3NP, UK

${ }^{34}$ Department of Physics, University of Wisconsin, River Falls, Wisconsin 54022, USA

${ }^{35}$ Oskar Klein Centre and Department of Physics, Stockholm University, SE-10691 Stockholm, Sweden

${ }^{36}$ Department of Physics and Astronomy, Stony Brook University, Stony Brook, New York 11794-3800, USA

${ }^{37}$ Department of Physics and Astronomy, University of Alabama, Tuscaloosa, Alabama 35487, USA

${ }^{38}$ Department of Astronomy and Astrophysics, Pennsylvania State University, University Park, Pennsylvania 16802, USA

${ }^{39}$ Department of Physics, Pennsylvania State University, University Park, Pennsylvania 16802, USA

${ }^{40}$ Department of Physics and Astronomy, Uppsala University, Box 516, S-75120 Uppsala, Sweden

${ }^{41}$ Department of Physics, University of Wuppertal, D-42119 Wuppertal, Germany

${ }^{42}$ DESY, D-15735 Zeuthen, Germany

(Received 21 February 2012; published 26 July 2012)

The first dedicated search for ultrahigh-energy (UHE) tau neutrinos of astrophysical origin was performed using the IceCube detector in its 22-string configuration with an instrumented volume of roughly $0.25 \mathrm{~km}^{3}$. The search also had sensitivity to UHE electron and muon neutrinos. After application of all selection criteria to approximately 200 live-days of data, we expect a background of $0.60 \pm$ 0.19 (stat) ${ }_{-0.58}^{+0.56}$ (syst) events and observe three events, which after inspection, emerge as being compatible with background but are kept in the final sample. Therefore, we set an upper limit on neutrinos of all flavors from UHE astrophysical sources at 90\% C.L. of $E_{\nu}^{2} \Phi_{90}\left(\nu_{\mathrm{x}}\right)<16.3 \times 10^{-8} \mathrm{GeV} \mathrm{cm}^{-2} \mathrm{sr}^{-1} \mathrm{~s}^{-1}$ over an estimated primary neutrino energy range of $340 \mathrm{TeV}$ to $200 \mathrm{PeV}$.

DOI: 10.1103/PhysRevD.86.022005

\section{INTRODUCTION}

Proposed astrophysical sources of observed ultrahighenergy (UHE) cosmic rays are expected to also produce ultrahigh-energy neutrinos, mainly via charged pion decay following interactions on ambient matter and radiation $[1,2]$. Candidate neutrino sources include active galactic nuclei, gamma-ray bursts and microquasars [3-5]. Neutrinos are expected to arrive at Earth with a flavor ratio of $\nu_{\mathrm{e}}: \nu_{\mu}: \nu_{\tau}=1: 1: 1$ in the standard neutrino oscillation scenario [6]. Other neutrino production and propagation models predict different flux ratios at Earth [7-9]. If there

\footnotetext{
*Physics Department, South Dakota School of Mines and Technology, Rapid City, SD 57701, USA

${ }^{\dagger}$ Also at Los Alamos National Laboratory, Los Alamos, NM 87545, USA

${ }^{*}$ Also at Sezione INFN, Dipartimento di Fisica, I-70126, Bari, Italy

\$ seo@fysik.su.se, corresponding author

"Also at NASA Goddard Space Flight Center, Greenbelt, MD 20771, USA
}

PACS numbers: 95.85.Ry, 14.60.Lm, 95.30.Cq, 95.55.Vj

are many astrophysical point sources of neutrinos, but each one is too weak to be distinguished individually from background, then a suitable detection strategy is to perform a cumulative search for "diffuse" flux of UHE neutrinos over the full available solid angle.

In previous searches [10-12], diffuse astrophysical UHE neutrinos were distinguished from atmospheric neutrinos by requiring the energy of candidate UHE neutrino events to exceed a certain threshold. In this work, we present techniques for identifying $\nu_{\tau}$ interactions and show the results of the first search for diffuse astrophysical UHE neutrinos, which specifically selected events consistent with several $\nu_{\tau}$ interaction topologies.

At $E_{\nu} \gtrsim 1 \mathrm{PeV}$, a search for UHE $\nu_{\tau}$ offers several advantages over UHE $\nu_{\mathrm{e}}$ and $\nu_{\mu}$ searches. Partially and fully contained interactions of UHE $\nu_{\tau}$ in the detector can produce very distinctive signatures owing to the macroscopic $\tau$ decay length. Each such signature should appear in proportion to the well-measured $\tau$ branching ratios [13], providing a useful cross-check on the positive identification 
of multiple $\nu_{\tau}$ events. As shown below, the chief sources of possible background events are unlikely to mimic these signatures. Also, at these energies, there is negligible intrinsic $\nu_{\tau}$ background in the conventional atmospheric neutrino flux [14]. The prompt $\nu_{\tau}$ flux from charm hadron decays in cosmic-ray-induced air showers is also expected to be small [15-17]. The majority of the signal $\nu_{\tau}$ is expected to come from the vicinity of the horizon since there is insufficient material for interactions in the downward-going direction and $\nu_{\tau}$ passing through the Earth emerge [18] at energies too low to create a UHE signature.

The $\nu_{\tau}$ event topology depends on how much of the event is contained in the detector, the $\nu_{\tau}$ energy, and the composition of the $\tau$ decay products. In this work, only nonmuonic $\tau$ decays were considered. A partially contained UHE $\nu_{\tau}$ having only the decay vertex of $\tau$ in the instrumented volume is denoted a "lollipop," while one having only the production vertex of the $\tau$ in the instrumented volume is denoted an "inverted lollipop." A fully

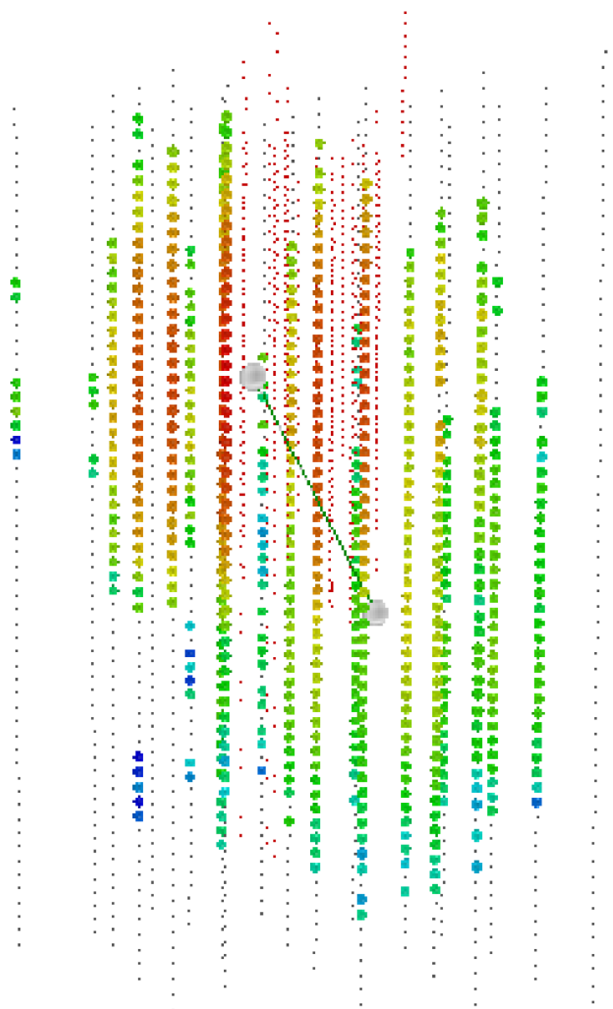

FIG. 1 (color online). A simulated double-bang event with a primary $\nu_{\tau}$ energy of $47 \mathrm{PeV}$ entering the IC22 detector at a zenith angle of $35^{\circ}$. The two bigger circles in gray color represent the vertices of tau creation (upper left) and decay (lower right) which are connected by a tau track. Each smaller circle represents a photomultiplier tube which observed light produced by the event. The denser PMTs in the upper middle belong to AMANDA and are not used in this analysis. The arrival times are indicated by colors which are ordered following the colors of the rainbow, with red corresponding to earlier times and violet to later times. contained UHE $\nu_{\tau}$ having both production and decay vertices well-separated in the instrumented volume is denoted a "double bang" [19]. Figure 1 shows a simulated double-bang event in the 22-string configuration of the IceCube detector (IC22) which had an instrumented volume of roughly $0.25 \mathrm{~km}^{3}$

Applying criteria to identify lollipop, inverted lollipop and double-bang signatures produced by $\nu_{\tau}$ interactions, we derived limits on the diffuse UHE neutrino flux. We assumed a flux ratio of $\nu_{\mathrm{e}}: \nu_{\mu}: \nu_{\tau}=1: 1: 1$ for this analysis. We used 282.4 live-days of data collected in 2007-2008 by IC22. We describe the IC22 detector in Sec. II and the experimental and simulated data samples in Sec. III. We present our analysis in Sec. IV and the results in Sec. V. We discuss systematic errors in Sec. VI and our conclusions in Sec. VII.

\section{THE ICECUBE 22-STRING DETECTOR}

The 22-string configuration of IC22 was deployed in early 2007, began taking physics-quality data in May of that year and ended at the transition to IceCube's 40-string configuration in April 2008. Each string consists of 60 digital optical modules (DOMs) buried deep in the icecap at the South Pole, with regular $17 \mathrm{~m}$ vertical spacing from 1450 to $2450 \mathrm{~m}$ below the surface, for a total of 1320 DOMs. The strings are situated on a regular grid with $125 \mathrm{~m}$ horizontal interstring spacing, covering the area shown in Fig. 2. Each DOM houses a photomultiplier tube (PMT) to detect the Cherenkov light, electronics for pulse digitization and other functions and remotelycontrollable calibration light sources. To reduce the impact of PMT signals due to random noise, only detected signals with minimum 0.25 single photoelectron (p.e.) PMT pulse height were digitized by two types of waveform digitizers in situ: the analog transient waveform digitizer (ATWD)

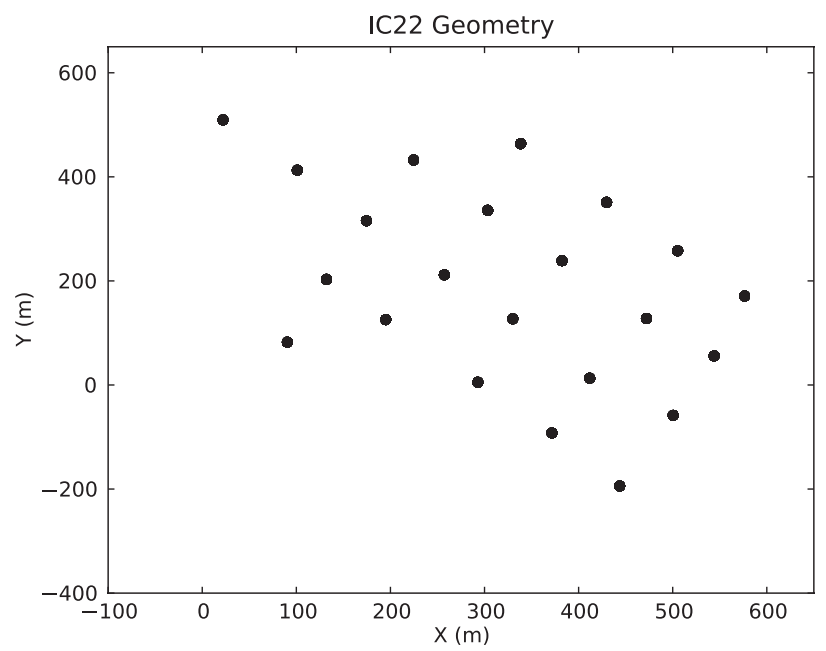

FIG. 2. Top view of the IceCube 22 string detector. Each string is represented as a dot. 
and a fast analog to digital converter (fADC). The time resolution of the ATWD (fADC) is about $3.33 \mathrm{~ns}(25 \mathrm{~ns}$ ) with a readout time window of about $450 \mathrm{~ns}(6.4 \mu \mathrm{s})$. Thus, the ATWD is used to capture detailed waveform information on a short time scale while the fADC records less detailed information on a longer time scale. The ATWD also supports three channels with different gains $(\times 16, \times 2$, and $\times 0.25$ ) to extend its effective dynamic range.

To further remove random noise, the digitized signal in a DOM was required to be in close temporal coincidence with a signal in neighboring DOMs. The signals satisfying such a temporal condition in hardware are called local coincidence (LC) hits. LC hits were then checked to see whether or not they satisfied a software-based trigger which selected for patterns potentially produced by a charged particle. Groups of hits which satisfied a trigger condition were packaged into "events." Higher-level "filter" algorithms were applied to each event, and those events passing one or more filter conditions were transmitted over satellite to the northern hemisphere for higher-level analysis. However, all the data satisfying the software trigger conditions were stored on tape and shipped to the northern hemisphere once a year. The software trigger and filter conditions applied to the data used in this analysis are described in the section below. For more detail on the design, construction and performance of IceCube in general, see Refs. [20-23] and references therein.

\section{DATA}

\section{A. Experimental data}

The DOM signals satisfying the LC condition were required by the online data acquisition system at the surface computing system in the IceCube Laboratory to satisfy a "simple majority trigger" condition under which eight or more DOMs reported signals in a $5 \mu \mathrm{s}$ time window ("SMT8"). The IC22 trigger rate of 500 to $620 \mathrm{~Hz}$ followed the seasonal variation in the cosmic-ray muon flux. The data acquisition system grouped together DOM hits satisfying the trigger condition into an event using a broadened $\pm 10 \mu$ s time window. Triggered events used in this analysis were accepted if they also satisfied the extremely high-energy (EHE) filter applied to the data online at the South Pole to reduce low-energy events consistent with background. The EHE filter required $\geq 80$ DOMs registering hits in the event.

We split off about $30 \%$ of the full IC22 data set $(82.4$ live-days, uniformly distributed in time across the datataking period) to use in conjunction with simulated data in the design of our subsequent selection criteria. In keeping with our procedures for maintaining blindness in the analysis of data, and thereby reducing human bias in the analysis of the data, the final result is based on the application of these selection criteria, unaltered, to the remaining $70 \%$ of the data set (200 live-days).

\section{B. Simulated data}

We employed simulated data to develop criteria which enhanced a possible astrophysical neutrino signal while diminishing backgrounds from atmospheric neutrinos and cosmic-ray muons. Exclusive use of simulated data also permitted us to maintain blindness. For the neutrinos, the All Neutrino Interaction Simulation package [24] was used and each neutrino flavor was produced separately. The neutrinos were generated following an $E^{-1}$ energy spectrum to enhance event statistics at higher energy where this analysis is sensitive. The neutrinos were propagated through the Earth where the Earth shadow effect [25] of neutrinos and $\nu_{\tau}$ regeneration [26,27] were taken into account in our simulation.

The events were then run through the IceCube detector simulation. The muon (electron) neutrinos were generated over all zenith angles in the energy range between 10 (50) $\mathrm{GeV}$ to $10 \mathrm{EeV}$ while tau neutrinos were generated between $1 \mathrm{TeV}$ and $1 \mathrm{EeV}$.

Cosmic-ray muon backgrounds were simulated by generating air shower events using the CORSIKA package [28], then propagating the muons to and through the detector volume with the MMC package [29] and finally applying the detector simulation to the resulting set of particles.

For solitary air showers, a two-component model [30] was used. In this model, the entire mass spectrum of cosmic rays is approximated by only proton and iron components. Compared to Hörandel's polygonato model [31], the two-component model agrees better with experimental data at higher energy (beyond $100 \mathrm{TeV}$ ) where this analysis is sensitive. The cosmic-ray primaries are sampled with an $E^{-2}$ spectrum. In this way, we were able to produce events more efficiently at the higher primary energies which contribute most strongly to the background at ultrahigh energies. The cosmic-ray flux was then reweighted to match the expected spectrum.

The acceptance of IC22 admits the possibility of detecting muons from multiple quasisimultaneous air-shower events, so we also simulated muons from two coincident airshower events. (Higher multiplicities occur at a negligible rate in IC22 and were not simulated.) For coincident air showers, Hörandel's polygonato model of cosmic rays was used. Solitary (coincident) atmospheric air showers were generated with energies between $10(0.6) \mathrm{TeV}-100 \mathrm{EeV}$ and zenith angles between $0-90^{\circ}$.

After event generation and detector simulation, the simulated data were processed in the same way as real data, i.e., with sequential applications of trigger and filter conditions, as described earlier.

\section{TAU NEUTRINO IDENTIFICATION}

\section{A. Selection criteria}

Based on the characteristics of simulated data, we formulated several event selection criteria to exploit the UHE 
$\nu_{\tau}$ signatures of a track plus one or two showers, in contrast to conventional pure tracklike or pure showerlike events. Two such criteria use the reconstructed total number of photoelectrons $\left(N_{\text {pe }}\right)$ per DOM. The time associated with such a multiphotoelectron deposit in each DOM is the time of the first reconstructed photoelectron it detected. Looking at the full event time window, $N_{\text {pe }}$ for each DOM is plotted vs time and denoted $N_{\mathrm{pe}}^{\mathrm{DOM}}(t)$. Figure 3 shows $N_{\mathrm{pe}}^{\mathrm{DOM}}(t)$ for a simulated inverted lollipop (top) and a simulated muon event (bottom). Note that the times of the hits are with respect to the event trigger time which has an extended readout time window of $\pm 10 \mu \mathrm{s}$ in IC22. For this reason, all the hit times exhibit at least a $10 \mu \mathrm{s}$ offset.

To exploit the power of $N_{\mathrm{pe}}^{\mathrm{DOM}}(t)$, we devised a parameter called "maximum current ratio" ( $\mathrm{IR}_{\max }$ ), defined as the maximum of $I_{\text {in }} / I_{\text {out }}$ where $I_{\text {in(out) }}=\Delta Q_{\text {in(out) }} / \Delta T_{\text {in(out) }}$. Here, $\Delta Q_{\text {in }}$ was the charge, measured in p.e., collected
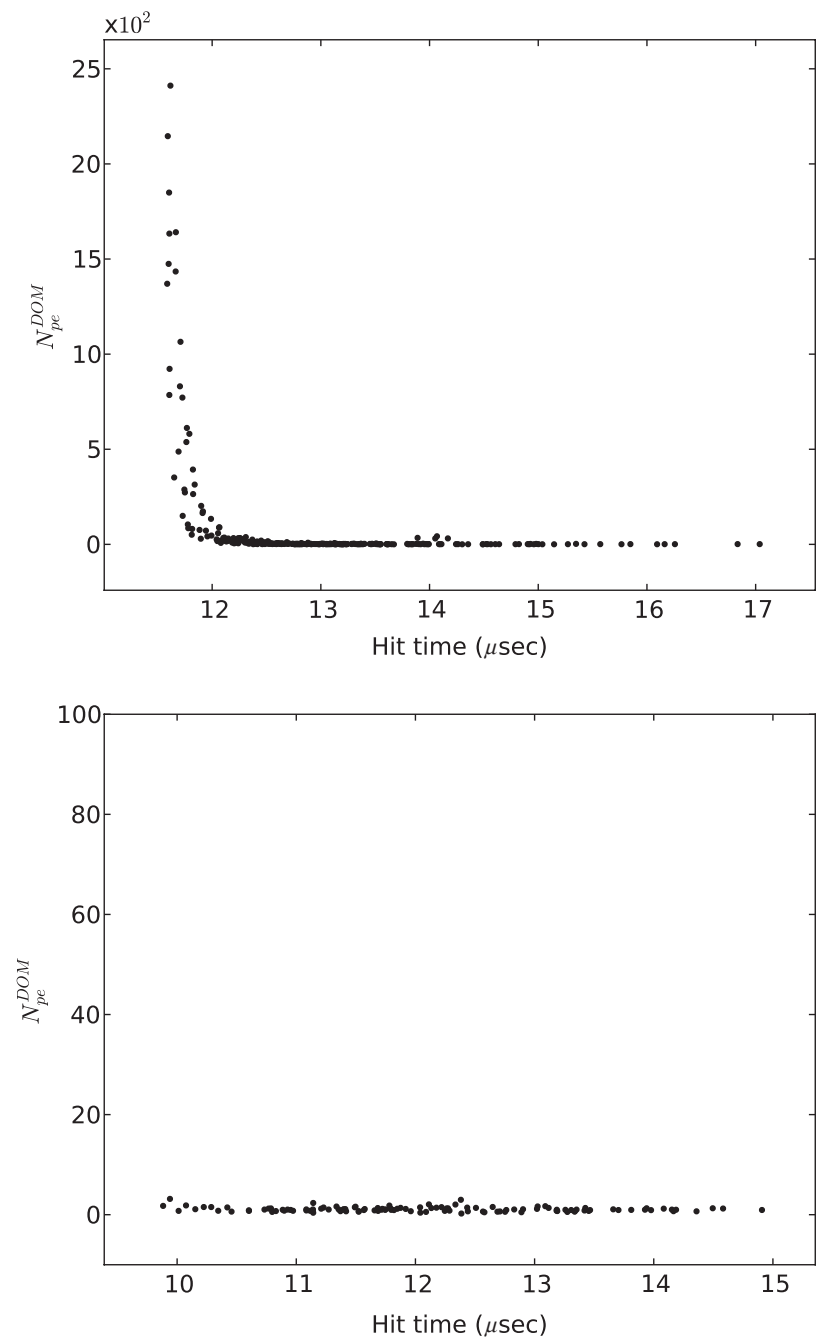

FIG. 3. The quantity $N_{\text {pe }}^{\mathrm{DOM}}(t)$ for a simulated inverted lollipop (top) and a simulated muon event (bottom), with primary particle energies of $25.4 \mathrm{PeV}$ and $2.38 \mathrm{PeV}$, respectively. The peak of the top plot is at roughly 2500 photoelectrons. by the DOMs in a sliding time window of length $\Delta T_{\text {in }}$. The time window was optimized in this analysis to be $1.2 \mu \mathrm{s}$ long. The corresponding "out" variables were the charge and time measured outside the sliding time window (see Fig. 4). As shown in Fig. 5, $\mathrm{IR}_{\max }$ is small for tracklike events and large for events containing showers, such as those produced by $\nu_{\tau}$. Since the $\mathrm{IR}_{\max }$ cut is related to energy, it will be applied to data as the last cut together with the other energy related cut explained at the end of this subsection.

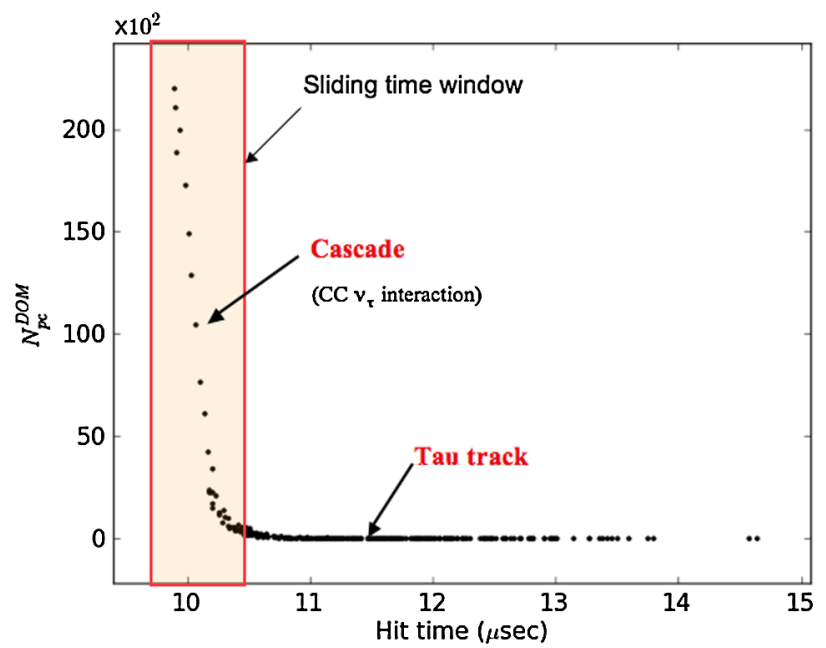

FIG. 4 (color online). The maximum current ratio $\left(\mathrm{IR}_{\max }\right)$ for an event is calculated by finding the maximum ratio of charge inside a sliding time window to the charge outside this window. This variable is expected to be larger for $\nu_{\tau}$ events (as in the example shown here) than for background events due to atmospheric muons.

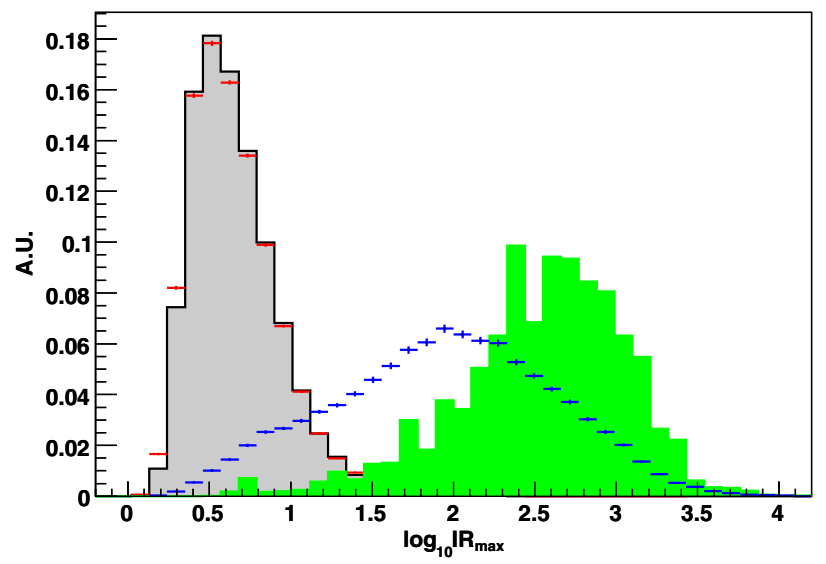

FIG. 5 (color online). The logarithm of the $\mathrm{IR}_{\max }$ parameter for simulated signal (green histogram for lollipop and blue points for all $\nu_{\tau}$ events) and background (red points for atmospheric muon) events and for data (gray histogram) passing the EHE filter. The distributions have been normalized to unit integrals to highlight the separation between signal and background. The $\mathrm{IR}_{\max }$ distributions of inverted lollipop and double-bang events are also well-separated from the background. 
Although $\mathrm{IR}_{\max }$ is very effective at distinguishing most simple tracklike background events from signal events, highly energetic muons can stochastically deposit large amounts of energy along their track lengths via bremsstrahlung, pair production or photonuclear interactions, potentially mimicking $\nu_{\tau}$ events. Figure 6 shows an example of simulated muon with such a bremsstrahlung whose $\mathrm{IR}_{\max }$ value could be similar to that of a $\nu_{\tau}$. Theoretically, $\nu_{\tau}$ events are most likely to have a large $N_{\mathrm{pe}}^{\mathrm{DOM}}(t)$ at one or both of the temporal edges of the event. In practice, $\nu_{\tau}$ events had a large $N_{\mathrm{pe}}^{\mathrm{DOM}}(t)$ in the earliest third due to the presence of highly scattered photons which extended the temporal edge of the event to much later times. We expect future analyses to be able to devise criteria which reduce the impact of these scattered photons.

The "local charge density" parameter $\rho_{q}$, with units of p.e./ns, was introduced to remove events consistent with a large energy deposit away from either temporal edge.

Partitioning each event into three equal time windows, we calculate the per-DOM ratios of charge to time in each window. These ratios are denoted $\rho_{q}(I), \rho_{q}(I I)$ and $\rho_{q}(I I I)$ in the first, second and third time window, respectively. Events for which $\rho_{q}(I)<5$ p.e. $/$ ns or $\rho_{q}(I I I)<5$ p.e. $/$ ns are rejected as being inconsistent with arising from a $\nu_{\tau}$ event, since $\nu_{\tau}$ are expected to make a significant energy deposition at the beginning and/or end of its interaction in the instrumented volume. Events with small $\rho_{q}(I I)$ are consistent with arising from $\nu_{\tau}$ and are not rejected. We expect future analyses to be able to devise criteria that reduce the impact of these scattered photons. Figure 7 shows $N_{\mathrm{pe}}^{\mathrm{DOM}}$ vs time and thus illustrates how $\rho_{q}$ can distinguish $\nu_{\tau}$ events from muon bremsstrahlung events. Figure 8 shows how well $\rho_{q}$ separates signal from background.

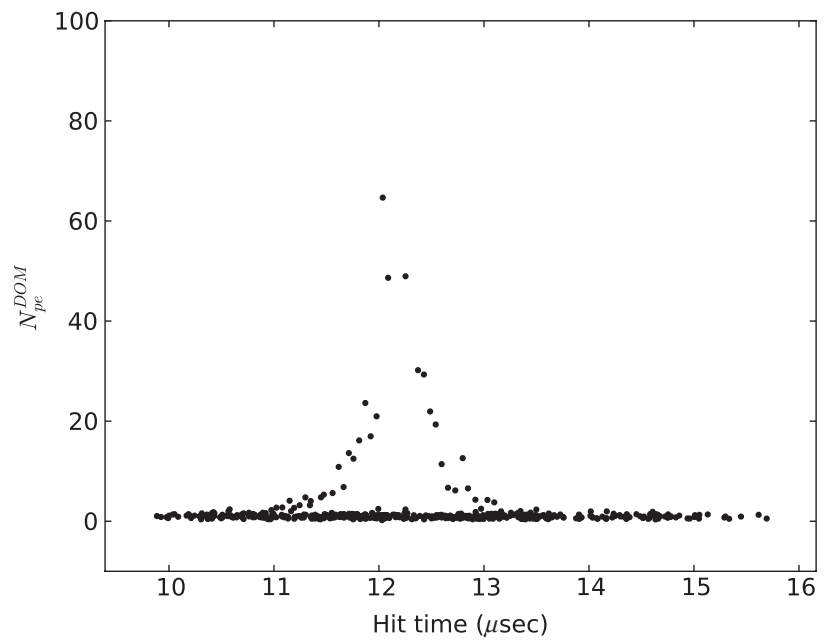

FIG. 6. The quantity $N_{\mathrm{pe}}^{\mathrm{DOM}}(t)$ for a simulated muon event, arising from a $336 \mathrm{PeV}$ cosmic-ray primary, with a high-energy bremsstrahlung energy loss.
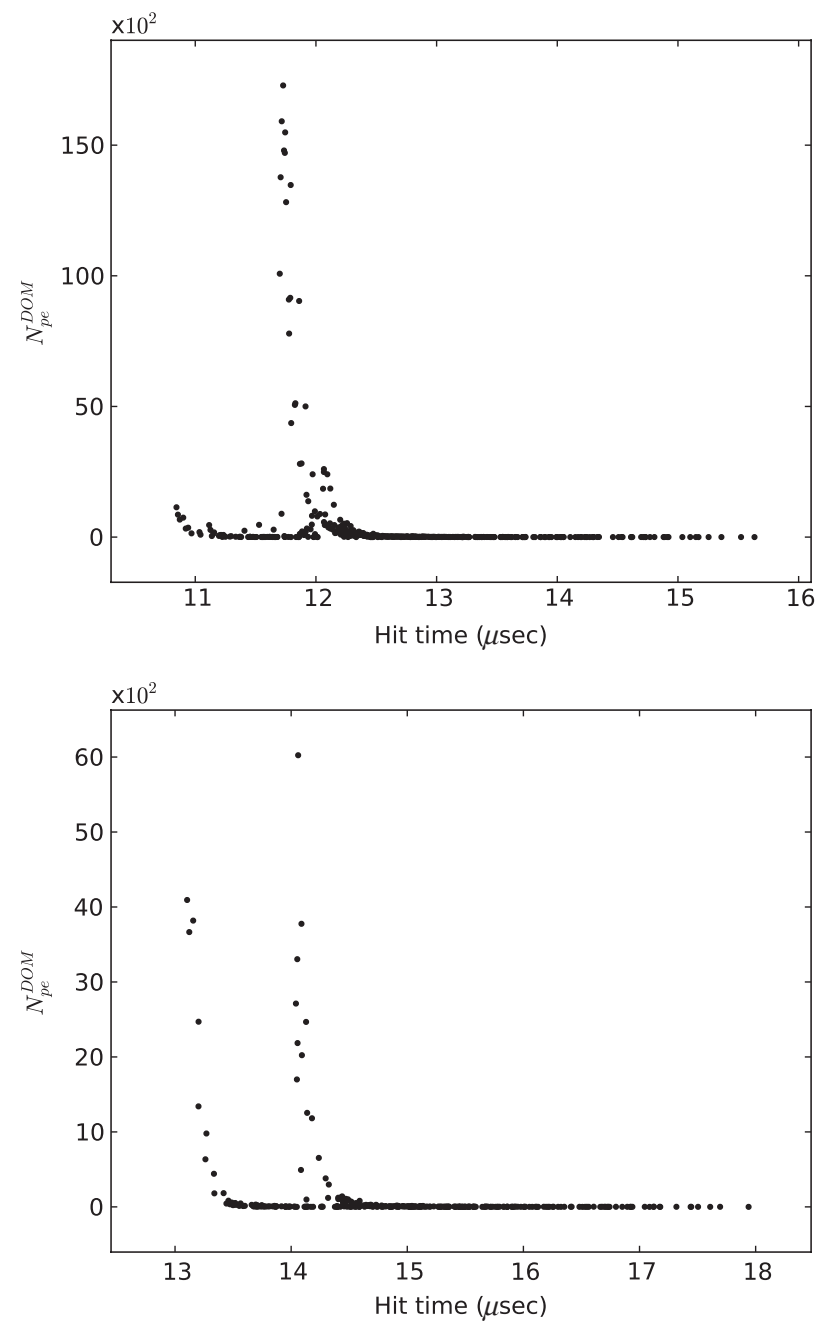

FIG. 7. The quantity $N_{\mathrm{pe}}^{\mathrm{DOM}}(t)$ for a simulated lollipop (top) and double-bang (bottom) event. The peak number of photoelectrons in the plots above ranges between roughly 6000 to over 15000 . These should be compared to $N_{\mathrm{pe}}^{\mathrm{DOM}}(t)$ for a simulated inverted lollipop in Fig. 3 (top) and for an atmospheric muon event in Fig. 6. The atmospheric muon, with a bremsstrahlung energy loss roughly in the middle of its contained track length, would be rejected by the cut on $\rho_{q}$ described in the text, whereas the lollipop, inverted lollipop and double bang would not because the bulk of the detected light occurs sufficiently early in the event.

Additional selection criteria were applied to further remove backgrounds. The flux of downward-going muons from cosmic-ray air shower events was reduced by implementing a "veto layer" in software, removing any events in which the average $Z$ position of the first 4 hits $\left(\bar{Z}_{\text {init }}\right)$ was in the top $50 \mathrm{~m}$ of the detection volume. Downward-going muons were further removed using the approximate event velocity $\bar{V}_{Z}(\mathrm{~m} / \mathrm{ns})$, constructed from the difference between the positions $Z_{\text {cog }}$ and $\bar{Z}_{\text {init }}$, divided by the difference in their respective times, i.e., $T_{\operatorname{cog}}$ and $\bar{T}_{\text {init }}$, where $Z_{\text {cog }}$ $\left(T_{\operatorname{cog}}\right)$ were the $Z$ position (time) of the center of gravity of 


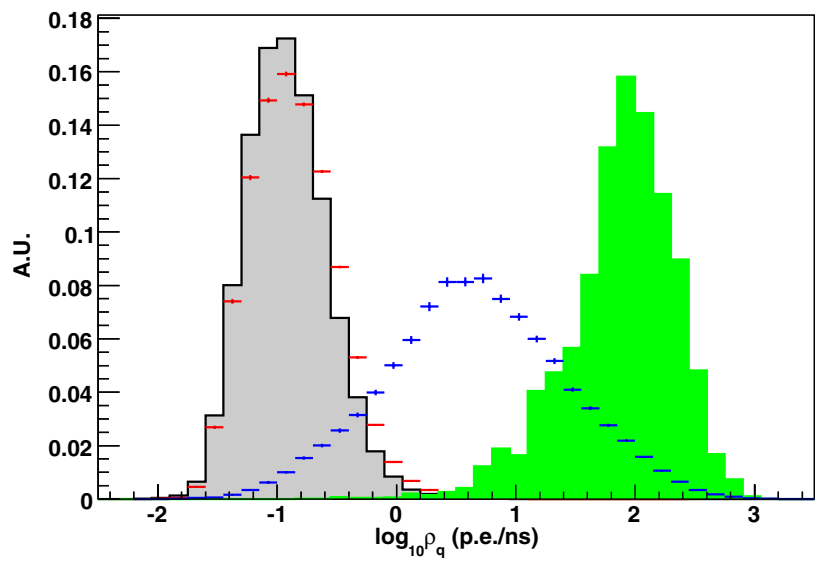

FIG. 8 (color online). The logarithm of the local charge density parameter $\left(\rho_{q}\right)$ for signal (green histogram for lollipop and blue points for all $\nu_{\tau}$ events) and background (red histogram for atmospheric muon) events, and for data (gray histogram) passing the EHE filter. The distributions have been normalized to unit integrals to highlight the separation between signal and background. The $\rho_{q}$ distributions of inverted lollipop and double-bang events are also well-separated from the background.

all hit DOMs. The times here are calculated using the average time of the hits used to calculate the $Z$ positions. We removed events consistent with a downward direction by requiring $\bar{V}_{Z}<-0.1 \mathrm{~m} / \mathrm{ns}$.

Background events arising from muon stochastic processes at or near the bottom of the detector, events whose muon tracks may go undetected, are removed by restricting our sample to events which were reasonably well-contained in the instrumented detector volume. We required the average depth position of all DOMs with signals to satisfy $Z_{\mathrm{cog}}>-330 \mathrm{~m}$ (as measured from the center of the detector).

We also applied a generic topological selection by calculating the eigenvalues of the tensor of inertia of pulse amplitudes (instead of conventional mass) [32] from hit DOMs of each event and keeping only those events that tended towards sphericity. Perfectly spherical events will have three equal tensor of inertia eigenvalues, while perfectly tracklike events will have one eigenvalue equal to zero. We therefore required that the ratio of smallest eigenvalue to the sum of all three eigenvalues was $>0.1$.

Remaining lower-energy events were further reduced in number by requiring a minimum $\mathrm{IR}_{\max }$ and $N_{\text {pe }}$ for each event. We required $\mathrm{IR}_{\max } \geq 200$ and $\log _{10} N_{\mathrm{pe}} \geq$ 4.2 , the values of which were based on an optimization which is described in the following section. Figure 9 shows the distributions of these two selection criteria for simulated signal, simulated background and $30 \%$ of the data, prior to the overall optimization of all the selection criteria.
The selection criteria described above are summarized in Table I.

\section{B. Optimization of selection criteria}

The final values for $\mathrm{IR}_{\max }$ and $N_{\mathrm{pe}}$ were optimized by minimizing the model rejection factor (MRF) [33] before applying them to the full data set. We varied the values of $\mathrm{IR}_{\text {max }}$ and $\log _{10} N_{\text {pe }}$ as shown in Fig. 10, finding a shallow minimum at $\mathrm{MRF} \sim 0.89$. At this MRF, the expected allflavor signal and background were 3.52 and 0.81 events, respectively, using the Waxman-Bahcall (WB) upper bound for signal, translated to account for what would be detected following standard neutrino oscillations, of $E_{\nu}^{2} \phi_{\nu}<\frac{3}{2} \times 4.5 \times 10^{-8} \mathrm{GeV} \mathrm{cm}^{-2} \mathrm{sr}^{-1} \mathrm{~s}^{-1}$ [4] for the signal neutrino flux normalization with $E^{-2}$ spectrum. Assuming standard neutrino oscillations, we expect onethird of this flux to be $\nu_{\tau}$. The corresponding optimized values are $\mathrm{IR}_{\max } \geq 300$ and $\log _{10} N_{\mathrm{pe}} \geq 4$.0. However, in order to be conservative in the face of limited simulated event statistics, we chose instead to use $\operatorname{IR}_{\max } \geq 200$ and $\log _{10} N_{\text {pe }} \geq 4.2$, resulting in an MRF $=0.92$ and expected all-flavor signal and background event counts of 3.18 and 0.60 , respectively.

\section{Signal selection efficiency}

The event rates for the selection criteria described in Sec. IVA were grouped into sets (EHE, S1-4) for reference purposes and are summarized in Table II for simulated signals. It is evident from Table II that this analysis, though designed to be sensitive primarily to UHE $\nu_{\tau}$ signals, also had appreciable sensitivity to UHE $\nu_{\mathrm{e}}$ and $\nu_{\mu}$ signals. The final limit described below will therefore be applicable to all neutrino flavors. Figures 11-14 show the distribution of event rates $(\mathrm{Hz})$ for each cut parameter for simulated signal as well as background and a sample of IC22 data. All plots show data after application of the EHE filter (Fig. 11) and sets of selection criteria S1 (Fig. 12), S2 (Fig. 13), and S3 (Fig. 14).

The efficiency of the event selection criteria for accepting signal can be obtained from Fig. 15 (top). The bottom plot of that same figure shows the effective area $A_{\text {eff }}$ for each neutrino flavor after application of the SMT8 trigger condition and the full suite of selection criteria. Using simulated signal, $A_{\text {eff }}$ is defined by $\Phi_{\nu} A_{\text {eff }} T=N_{\text {det }}$, where $\Phi_{\nu}$ is the neutrino flux prior to any propagation or interaction effects in the Earth, $T$ is a length of time, and $N_{\text {det }}$ is the number of detected events. The $A_{\text {eff }}$ is not used in the calculation of our limit on UHE neutrino production, but event rates for a particular theoretical model subject to the selection criteria in this analysis may be estimated via the product of the effective area and the model's predicted flux. In the energy range pertinent to this analysis, signal events must be either downward-going or horizontal due to Earth absorption of upward-going neutrinos for $E_{\nu}>\sim 100 \mathrm{TeV}$. 

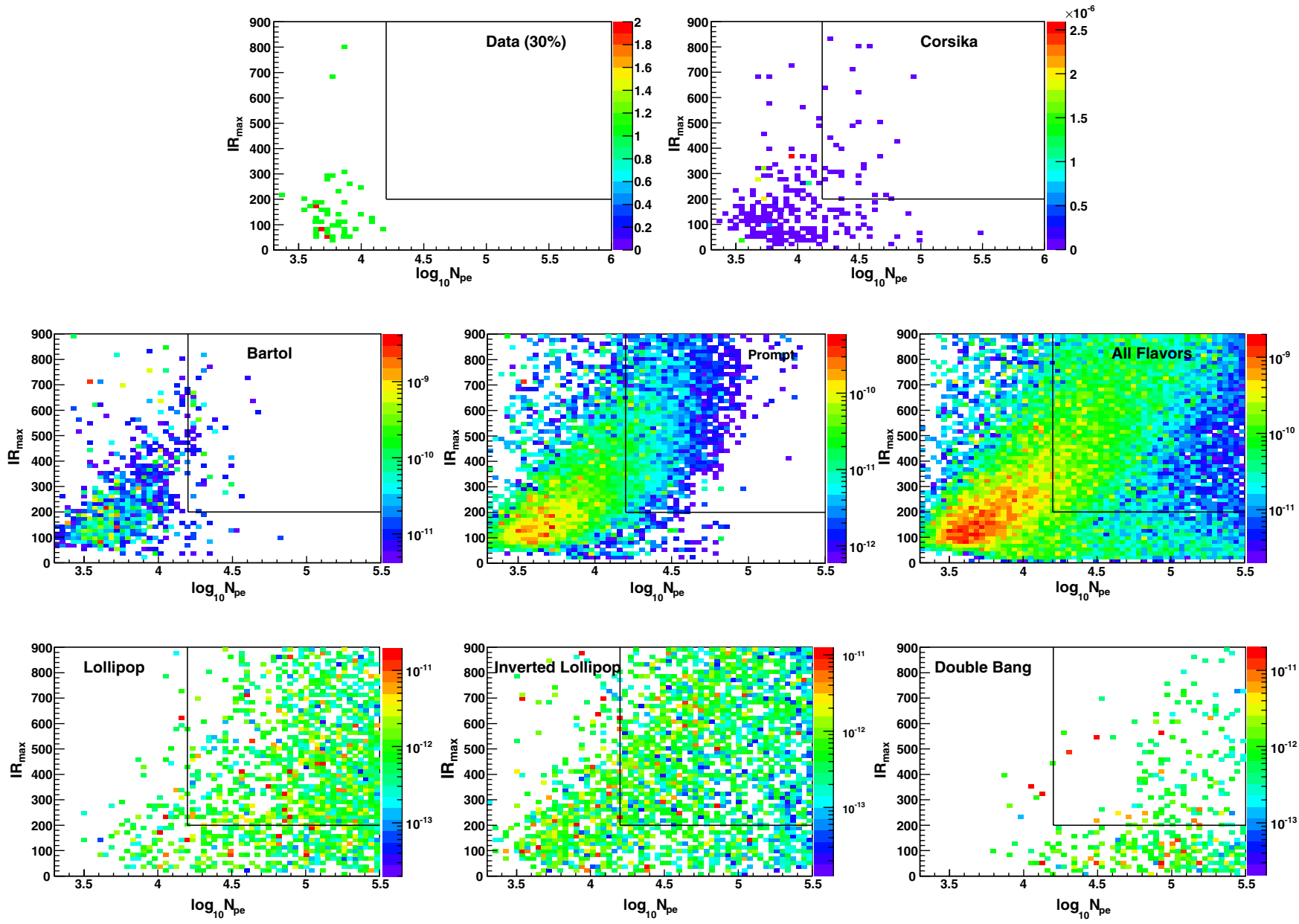

FIG. 9 (color online). Distributions of the quantities $\mathrm{IR}_{\max }$ vs $\log _{10} N_{\text {pe }}$ for $30 \%$ of the data (row 1, left), simulated cosmic-ray background (row 1, right), simulated atmospheric neutrinos (row 2, left), simulated prompt atmospheric neutrinos (row 2, middle), simulated all-flavor neutrino signal (row 2, right) and $\nu_{\tau}$ lollipop (row 3, left), inverted lollipop (row 3, middle) and double bang (row 3 , right), assuming an $E^{-2}$ spectrum and prior to the final selection criteria optimization. The color code represents the event rate in $\mathrm{Hz}$ except for the data where it represents number of events in $30 \%$ of the data sample (82.4 live-days). The region in the upper right of each plot, indicated by the black lines, designates the region selected by the optimized criteria as described in Sec. IV B.

\section{Background selection efficiency}

The event rates for simulated background and $30 \%$ of the data sample are summarized in Table III. Figures 11-14 show the distribution of event rates for background. The efficiency of the event selection criteria for rejecting background can be obtained from Fig. 15 (top), where the simulated background and $30 \%$ of the data sample match well at each cut level.

TABLE I. Summary of the selection criteria used in this analysis.

\begin{tabular}{ll}
\hline \hline Selection criterion: & \multicolumn{1}{c}{ Purpose: } \\
\hline NDOM $>80$ & Selects high-energy events which produce light in many DOMs. \\
$\rho_{q}(I), \rho_{q}(I I I)>5$ p.e. $/ \mathrm{ns}$ & Selects events creating light at beginning and/or end of event. \\
$\bar{Z}_{\text {init }}<450 \mathrm{~m}$ & Removes events with initial light depositions high in the detector. \\
$\bar{V}_{Z}<-0.1 \mathrm{~m} / \mathrm{ns}$ & Removes events consistent with downward trajectories. \\
$Z_{\mathrm{cog}}>-330 \mathrm{~m}$ & Selects well-contained events and removes cosmic-ray muons \\
& interacting near or below the bottom of the detector. \\
$\mathrm{ToI}>0.1$ & Favors events with more spherical than tracklike topologies. \\
$\mathrm{IR}_{\max } \geq 200$ & Selects events with large instantaneous light depositions. \\
$\log _{10} N_{\mathrm{pe}} \geq 4.2$ & Selects high-energy events which produce a large amount of light. \\
\hline \hline
\end{tabular}




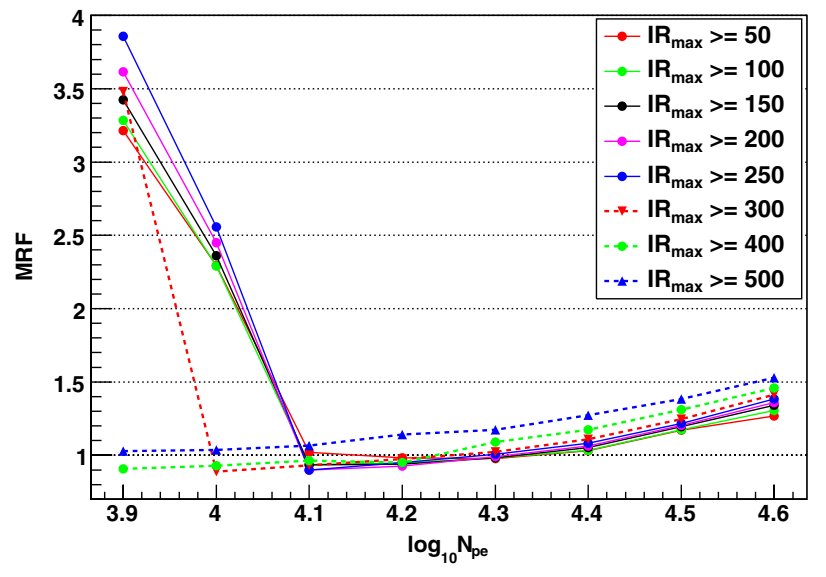

FIG. 10 (color online). We optimized the selection criteria for $\mathrm{IR}_{\max }$ and $\log _{10} N_{\mathrm{pe}}$ using the MRF formalism. The plot shows how the MRF varies as a function of $\log _{10} N_{\text {pe }}$ for different values of $\mathrm{IR}_{\max }$. We chose values for these parameters near but not exactly at the minimum shown for reasons explained in the text.

Figure 16 shows the distributions of the true zenith angle (top) and primary neutrino energy (bottom) from the simulation for the events which passed all the selection criteria. As expected, most $\nu_{\tau}$ were from near the horizon, with the angular acceptance peaking at about $100^{\circ}$ from vertical.

\section{RESULTS}

After unblinding the remaining 200 live-days of data and applying all the selection criteria, three events remained in the data sample. The predicted background from all simulated sources was $0.60 \pm 0.19$ events. The remaining data events are shown in Fig. 17.

From a detailed study of these events, we determined that one was consistent with light produced by an AMANDA optical module observed to emit light intermittently (Fig. 17, top). A second event was qualitatively consistent with background from a nearly horizontal muon interacting near the bottom of the detector (Fig. 17, middle). The third event had the characteristics of a neutrino-induced shower (Fig. 17, bottom), and was also in the final sample of an independent IC22 analysis which searched for showerlike signals [34]. However, we cannot rule out this event as being produced by a cosmic-ray
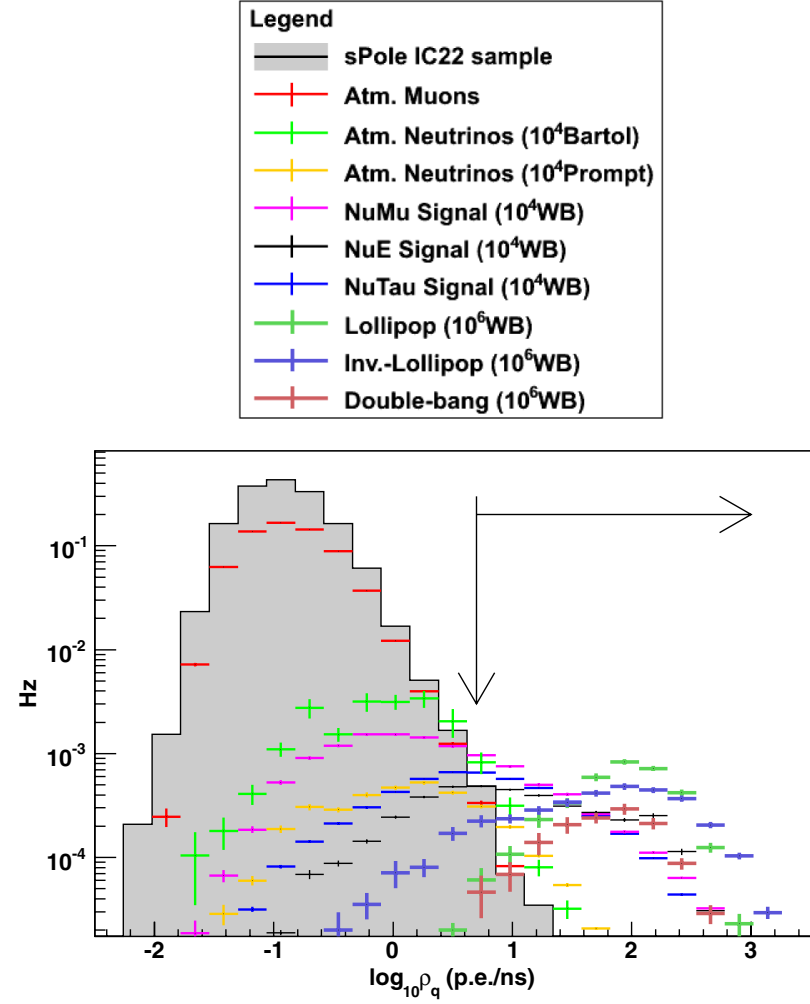

FIG. 11 (color online). Distributions of local charge density for signal, background (BG), and $30 \%$ of the IC22 data just before application of the "S1" set of selection criteria. The vertical (horizontal) arrow line represents the cut value (selected region) of that set.

muon accompanied by a stochastic high-energy bremsstrahlung energy-loss process. We have conservatively included all three events in the final sample in the derivation of the final result.

\section{SYSTEMATIC AND STATISTICAL ERRORS}

The systematic and statistical errors in this analysis were obtained using signal and background simulations and are summarized in Table IV. In the following subsections, systematic errors on signal and background are explained followed by our result including both errors.

TABLE II. Predicted signal event rates with statistical error after application of each set of selection criteria. LP, ILP and DB represent lollipop, inverted lollipop and double-bang, respectively. For signal rates, the flux was normalized to the WB bound. (The first column provides labels for reference purposes in subsequent tables and figures.)

\begin{tabular}{|c|c|c|c|c|c|c|c|}
\hline \multirow[t]{2}{*}{ Set No. } & \multirow[t]{2}{*}{ Selection Criteria } & \multicolumn{6}{|c|}{$\begin{array}{c}\text { MC simulation } \\
\text { Signal } \nu\left(E^{-2}\right)\end{array}$} \\
\hline & & $\mathrm{LP} \times 10^{-9}[\mathrm{~Hz}]$ & $\mathrm{ILP} \times 10^{-9}[\mathrm{~Hz}]$ & $\mathrm{DB} \times 10^{-9}[\mathrm{~Hz}]$ & $\nu_{\tau} \times 10^{-8}[\mathrm{~Hz}]$ & $\nu_{\mu} \times 10^{-8}[\mathrm{~Hz}]$ & $\nu_{e} \times 10^{-8}[\mathrm{~Hz}]$ \\
\hline $\mathrm{EHE}$ & $\mathrm{NDOM}>80$ & $3.48 \pm 0.11$ & $3.54 \pm 0.09$ & $4.45 \pm 0.16$ & $50.5 \pm 0.5$ & $119 \pm 2.2$ & $39.9 \pm 0.7$ \\
\hline $\mathrm{S} 1$ & $\rho_{q}(I), \rho_{q}(I I I)>5$ p.e. $/ \mathrm{ns}$ & $3.42 \pm 0.11$ & $3.05 \pm 0.08$ & $4.30 \pm 0.16$ & $24.0 \pm 0.3$ & $29.3 \pm 0.8$ & $23.9 \pm 0.6$ \\
\hline $\mathrm{S} 2$ & $\bar{Z}_{\text {init }}<450 \mathrm{~m}, \bar{V}_{Z}<-0.1 \mathrm{~m} / \mathrm{ns}$ & $2.55 \pm 0.10$ & $2.91 \pm 0.08$ & $3.95 \pm 0.16$ & $22.6 \pm 0.3$ & $24.9 \pm 0.8$ & $22.9 \pm 0.5$ \\
\hline S3 & $Z_{\mathrm{cog}}>-330 \mathrm{~m}, \mathrm{ToI}>0.1$ & $2.32 \pm 0.10$ & $2.29 \pm 0.08$ & $3.02 \pm 0.14$ & $15.7 \pm 0.3$ & $11.8 \pm 0.6$ & $17.5 \pm 0.5$ \\
\hline S4 & $\mathrm{IR}_{\max } \geq 200, \log _{10} N_{\mathrm{pe}} \geq 4.2$ & $1.72 \pm 0.08$ & $1.72 \pm 0.06$ & $2.07 \pm 0.11$ & $5.63 \pm 0.08$ & $3.70 \pm 0.15$ & $9.08 \pm 0.2$ \\
\hline
\end{tabular}



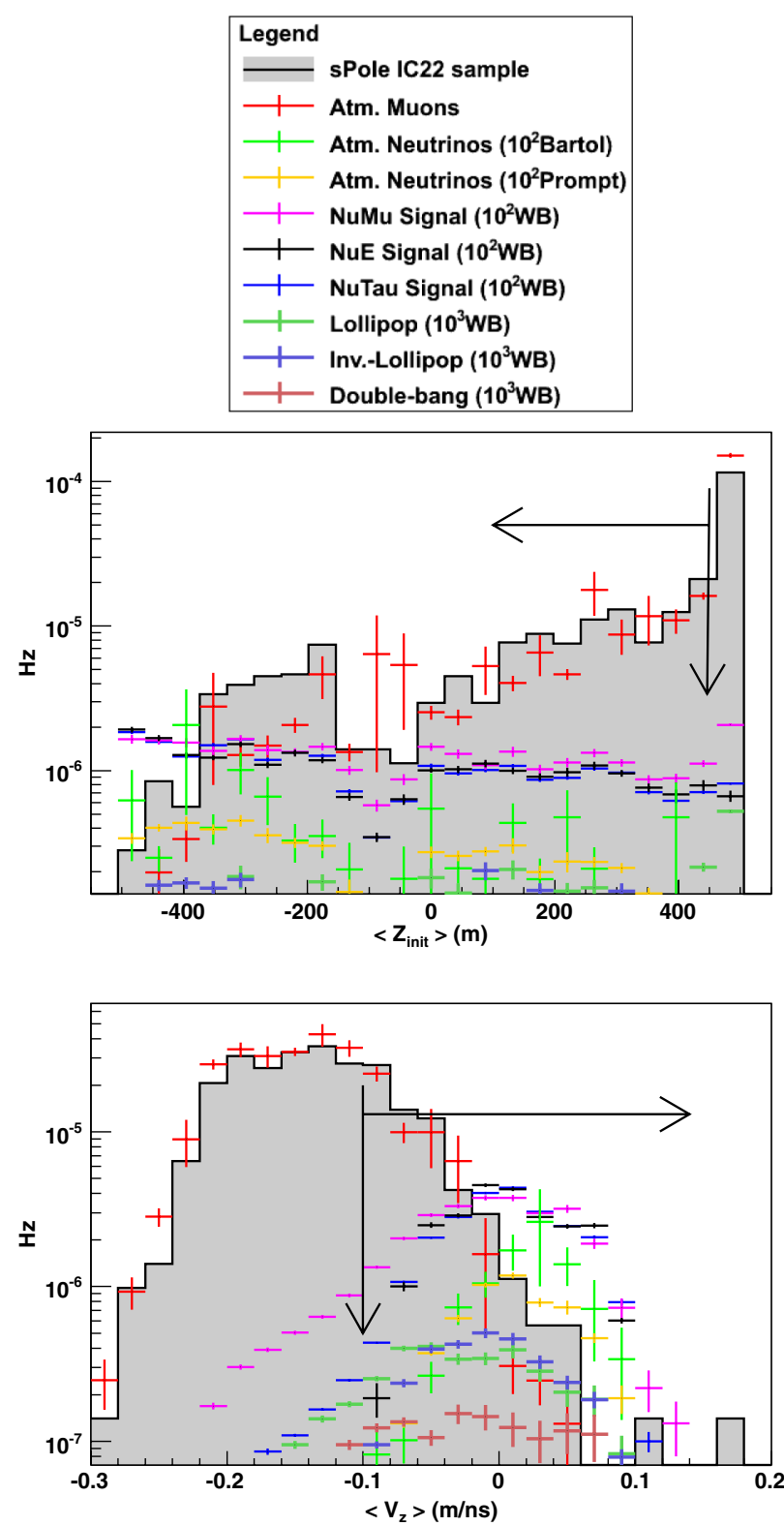

FIG. 12 (color online). Distributions of the average $z$-position of initial hits (top) and the average velocity $z$-component (bottom) for signal, BG, and $30 \%$ of the IC22 data just before application of the "S2" set of selection criteria. The vertical (horizontal) arrow lines represent the cut values (selected regions) of that set.

\section{A. Systematic errors for signal}

The systematic error due to our lack of precise knowledge of the DOM sensitivity to photons was obtained by simulating the effect of setting it to $90 \%$ and $110 \%$ of its nominal value resulting in $[-4.7 \%,+7.9 \%]$ error. The systematic error in the event rates reflecting uncertainties on the optical properties of the ice was obtained by simulating events using different ice models. The ice models were created from data generated using in situ light sources. The baseline ice model [35] for this analysis
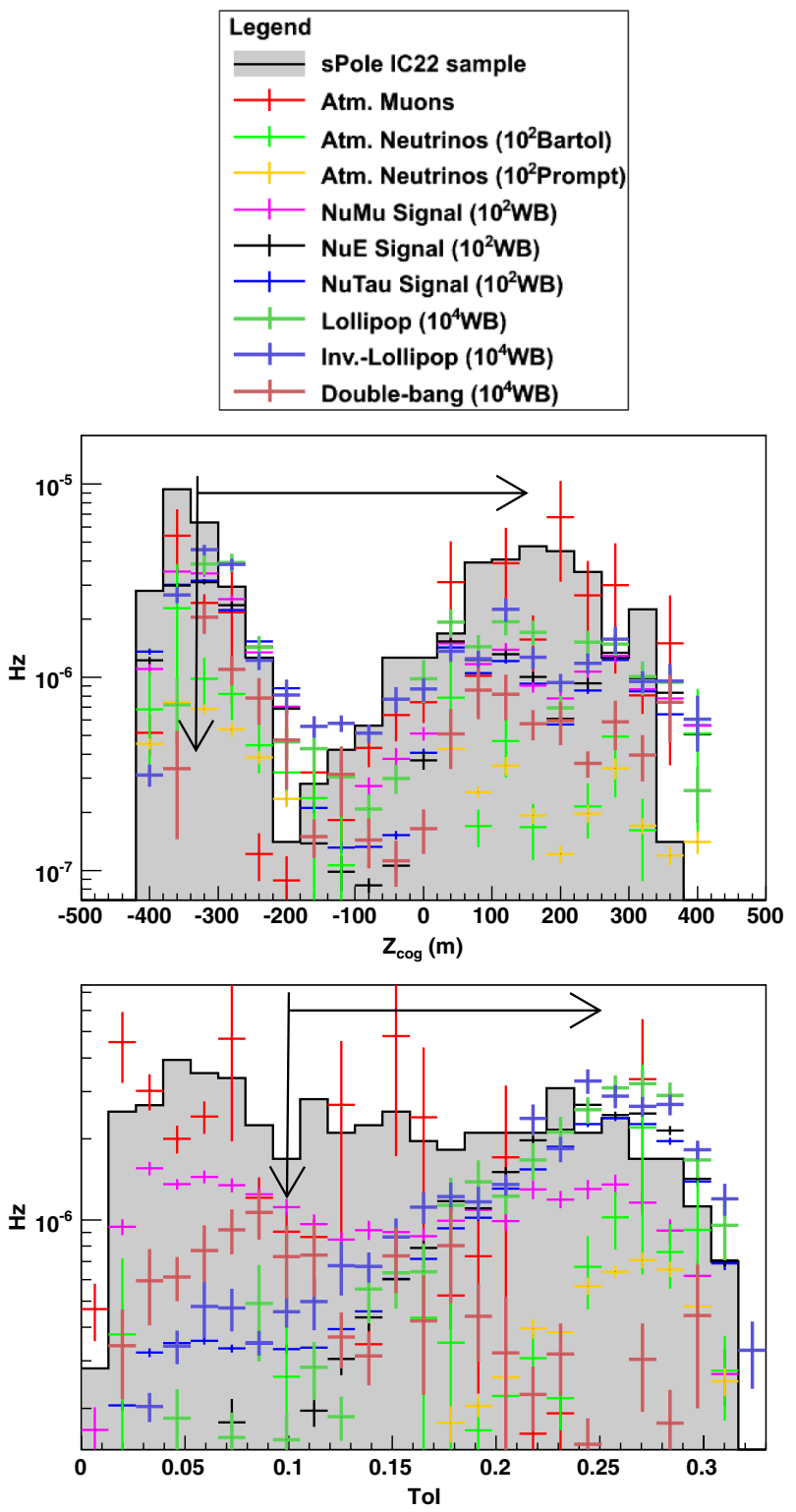

FIG. 13 (color online). Distributions of the center of gravity of the $z$-position (top) and tensor of inertia (bottom) for signal, BG, and $30 \%$ of the IC 22 data just before application of the "S3" set of selection criteria. The vertical (horizontal) arrow lines represent the cut values (selected regions) of that set.

used optical properties of the ice measured at AMANDA depths and extrapolated to IceCube depths, while an alternative ice model [36] obtained them with a direct fit to the full range of IceCube light source data. Comparing the predictions of the two ice models resulted in a $+29.4 \%$ error.

The systematic uncertainty in the neutrino cross section came from two sources. One was from theoretical uncertainty in the parton distribution function evaluation and structure function and the other was from errors in the experimental measurement of the parton distribution function by HERA [37]. From these two sources, we estimated 

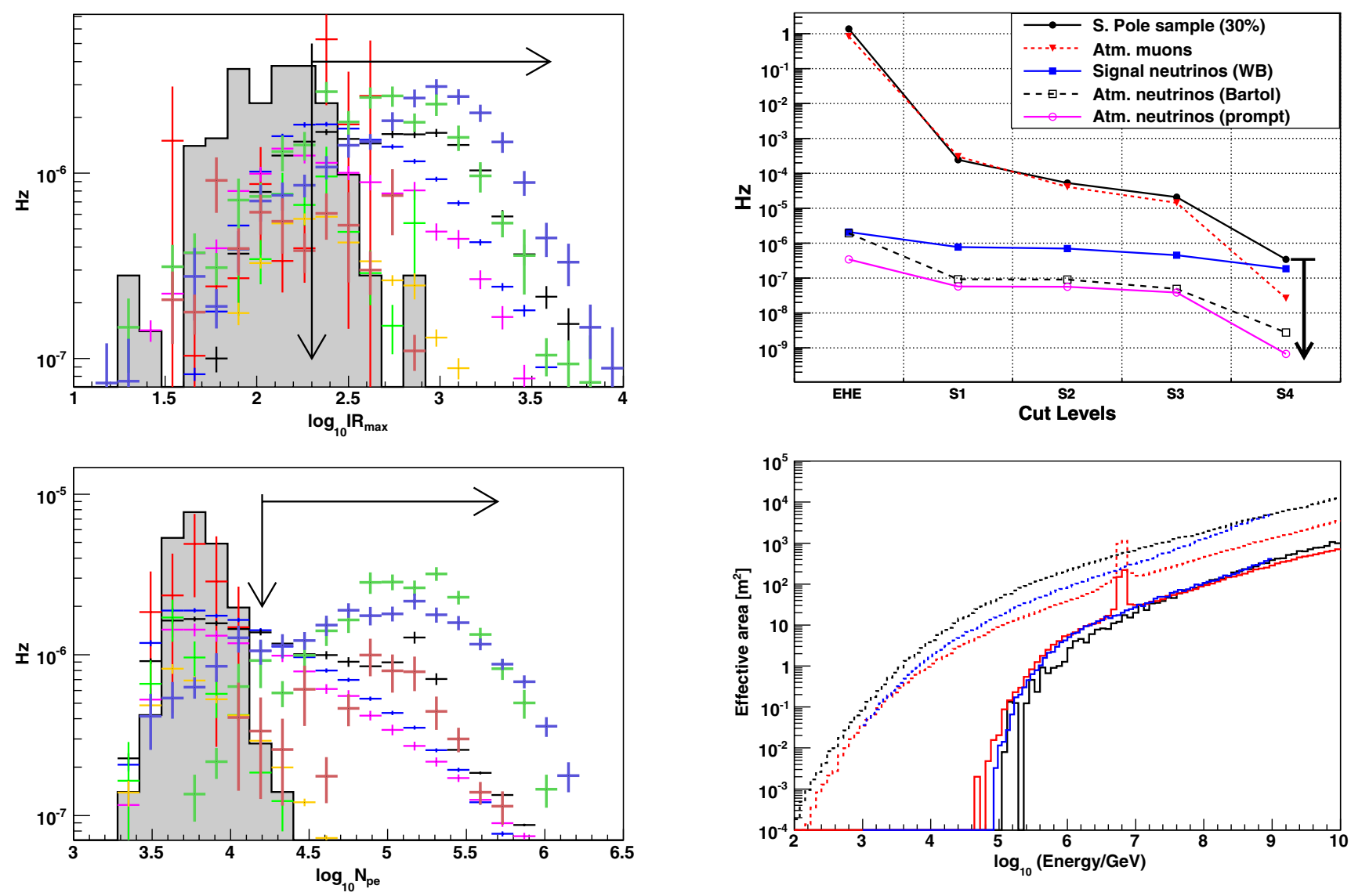

FIG. 14 (color online). Distributions of the maximum current ratio (top) and the number of photoelectrons (bottom) for signal, BG, and $30 \%$ of the IC22 data just before application of the "S4" set of selection criteria. The vertical (horizontal) arrow lines represent the cut values (selected regions) of that set. The legend for these plots is the same as in Fig. 13.

the systematic error in the neutrino cross section as $\pm 6.4 \%$. Very high-energy events could saturate PMTs by exceeding the PMT's dynamic range. This could result in an incorrect estimation of the original neutrino energy. Since the observable quantity most closely related to the energy is $N_{\text {pe }}$, the systematic error associated with the PMT saturation was obtained by observing the impact of changing the $N_{\mathrm{pe}}$ cut from $90 \%$ to $110 \%$ of its original value. This error was found to be $[-5.7 \%,+5.0 \%]$.

FIG. 15 (color online). Top: Event rate $(\mathrm{Hz})$ at each cut level for simulated signal and background, and $30 \%$ of the data sample. At S4, there were zero events in the data sample, and so the $90 \%$ C.L. upper limit value was plotted as indicated by the black arrow. Bottom: IC22 effective areas vs neutrino energy for each neutrino flavor (red: $\nu_{\mathrm{e}}$, blue: $\nu_{\tau}$, black: $\nu_{\mu}$ ) after application of the SMT8 trigger (dashed lines) and after application of all selection criteria (solid lines).

\section{B. Systematic errors for background}

The systematic errors due to uncertainties in DOM sensitivity, ice properties and DOM saturation behavior were obtained in the same manner as for the signals, as described in Sec. VI A. They were estimated as $[-4.7 \%$, $+7.9 \%],[-62 \%,+85 \%]$ and $[-28.9 \%,+5.3 \%]$, respectively.

TABLE III. Predicted background event rates with statistical error after application of each set of selection criteria. For conventional neutrinos (labeled "conv" in the table), the Bartol model [14] was used. For prompt neutrinos, the Martin's Golec-Biernat-Wusthoff (GBW) model [17] was used for $\nu_{\tau}$ and the Sarcevic standard model [16] was used for $\nu_{\mu}$ and $\nu_{e}$.

\begin{tabular}{|c|c|c|c|c|c|c|c|}
\hline \multirow[t]{2}{*}{ Set No. } & \multicolumn{5}{|c|}{ MC simulation } & \multirow[b]{2}{*}{$\begin{array}{l}\text { Background } \mu \\
\times 10^{-6}[\mathrm{~Hz}]\end{array}$} & \multirow{2}{*}{$\begin{array}{c}\text { Data } \\
30 \% \text { sample } \\
\times 10^{-6}[\mathrm{~Hz}]\end{array}$} \\
\hline & $\nu_{\mu}^{\text {conv }} \times 10^{-8}[\mathrm{~Hz}]$ & $\nu_{e}^{\text {conv }} \times 10^{-8}[\mathrm{~Hz}]$ & $\begin{array}{c}\text { Background } \nu \\
\nu_{\tau}^{\text {prompt }} \times 10^{-10}[\mathrm{~Hz}]\end{array}$ & $\nu_{\mu}^{\text {prompt }} \times 10^{-8}[\mathrm{~Hz}]$ & $\nu_{e}^{\text {prompt }} \times 10^{-8}[\mathrm{~Hz}]$ & & \\
\hline EHE & $184 \pm 14.0$ & $6.88 \pm 0.26$ & $33.4 \pm 0.4$ & $23.6 \pm 0.50$ & $9.95 \pm 0.13$ & 830000 & $1.37 \times 10^{6} \pm 438$ \\
\hline S1 & $8.21 \pm 1.80$ & $0.96 \pm 0.06$ & $9.74 \pm 0.17$ & $2.19 \pm 0.12$ & $3.46 \pm 0.05$ & 303 & $246 \pm 5.9$ \\
\hline S2 & $8.11 \pm 1.80$ & $0.96 \pm 0.06$ & $9.62 \pm 0.17$ & $2.05 \pm 0.12$ & $3.42 \pm 0.05$ & 41.2 & $53.3 \pm 2.7$ \\
\hline S3 & $4.16 \pm 0.66$ & $0.70 \pm 0.06$ & $7.12 \pm 0.14$ & $1.26 \pm 0.09$ & $2.55 \pm 0.04$ & 14.4 & $20.8 \pm 1.7$ \\
\hline S4 & $0.24 \pm 0.06$ & $0.04 \pm 0.003$ & $0.91 \pm 0.03$ & $0.15 \pm 0.02$ & $0.43 \pm 0.01$ & $0.026 \pm 0.01$ & 0 \\
\hline
\end{tabular}



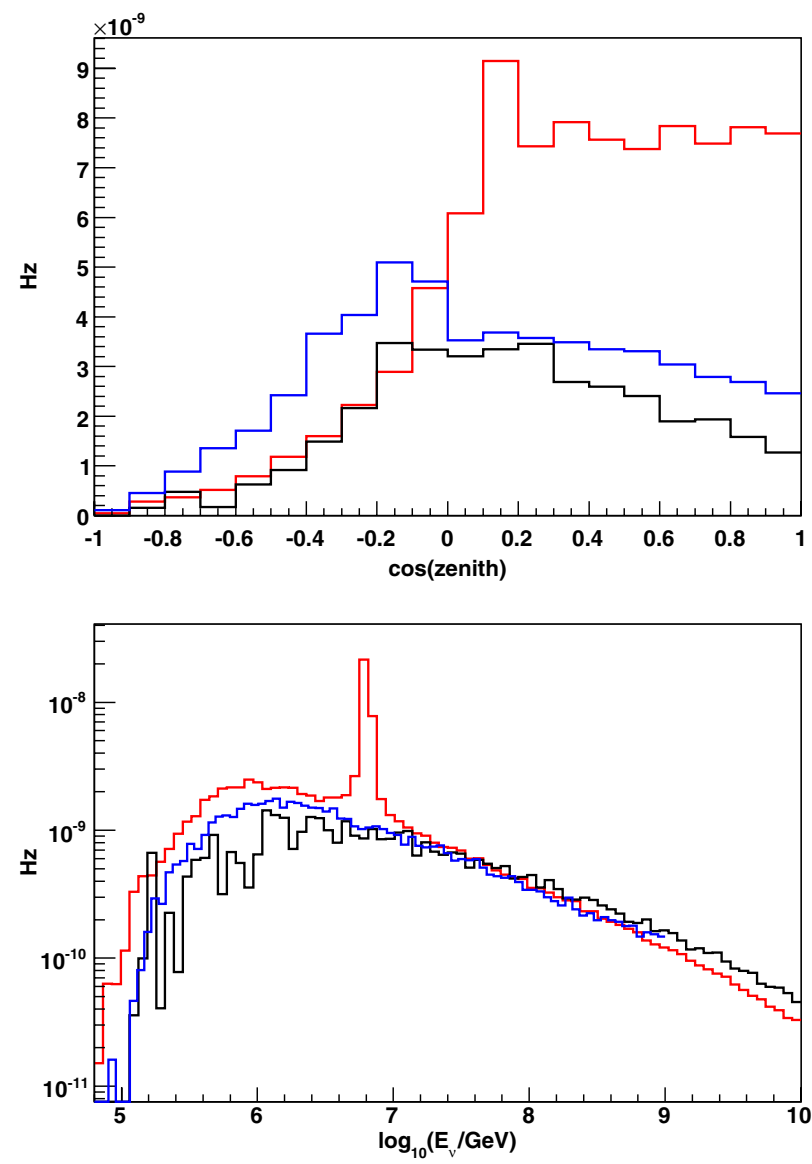

FIG. 16 (color online). The distribution of the true zenith angle (top) and the primary neutrino energy (bottom) from the simulation for the events passing all selection criteria. The primary neutrino energies in the $x$-axis were weighted to $E^{-2}$ spectrum. Red, blue and black lines correspond to $\nu_{\mathrm{e}}, \nu_{\tau}$ and $\nu_{\mu}$, respectively.

In addition, there are systematic errors which applied only to the background. The muon event rate is known to change as a function of the atmospheric temperature above the South Pole plateau. Since our muon simulation assumed a rate pegged to that seen in October, the seasonal variation was taken into account as a systematic error and was estimated as $[-24 \%,+18 \%]$ when compared with IC22 data at EHE filter level. The systematic error due to cosmic-ray composition was also obtained by switching constants and slopes between proton and iron in the twocomponent model data. At S3, just before the final cut, to have enough statistics, we obtained $-24 \%$ by this method.

There are alternative models for the prompt neutrino flux. For this analysis, the base models used for the prompt neutrino flux are Sarcevic standard flux model for $\nu_{\mu}$ and $\nu_{\mathrm{e}}$ [16] and Martin's Golec-Biernat-Wusthoff model for $\nu_{\tau}$ [17]. As an alternative, we have also considered the Sarcevic minimum and maximum flux models [16], from which we estimate a $[-59 \%,+30 \%]$ systematic error on the prompt neutrino flux.
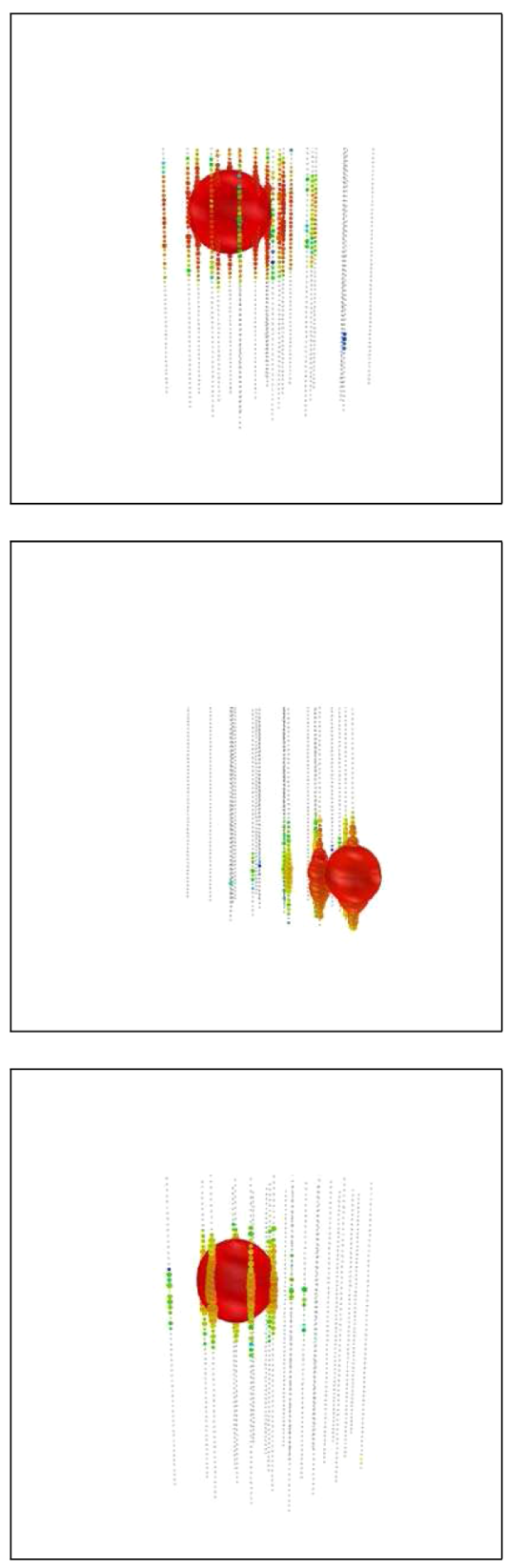

FIG. 17 (color online). Diagrams of the three events surviving the final selection criteria applied to the 200 live-days of IC22 data. The radius of each circle is proportional to the number of photons detected by the PMT.

\section{Result including statistical and systematic errors}

Since it was computationally feasible to generate a large amount of simulated signal, the statistical error on the simulated signal is small $( \pm 2.3 \%)$. By contrast, the considerably larger statistical error on the simulated background $( \pm 32 \%)$ reflects the aggregate effect of the high rejection efficiency of our selection criteria and the limitations imposed by finite computational resources. In summary, the expected signal and background events for 200 live-days with IC22 are $3.18 \pm 0.07$ (stat) ${ }_{-3.08}^{+2.99}$ (syst) and 
TABLE IV. Summary of the systematic and statistical errors for signal and background events from the simulated data.

\begin{tabular}{lcc}
\hline \hline Source & Signal & Background \\
\hline DOM sensitivity & $-4.7 \%,+7.9 \%$ & $-4.7 \%,+7.9 \%$ \\
Ice properties & $-0 \%,+29 \%$ & $-62 \%,+85 \%$ \\
$\nu$ cross section & $-6.4 \%,+6.4 \%$ & $\ldots$ \\
PMT saturation & $-5.7 \%,+5.0 \%$ & $-29 \%,+5.3 \%$ \\
Cosmic-ray flux & $\ldots$ & $-0 \%,+16 \%$ \\
Cosmic-ray composition & $\ldots$ & $-24 \%,+0 \%$ \\
Seasonal variation & $\ldots$ & $-24 \%,+18 \%$ \\
Prompt $\nu$ flux model & $\ldots$ & $-59 \%,+30 \%$ \\
Total Systematic error & $-7.9 \%,+31 \%$ & $-97 \%,+94 \%$ \\
Total Statistical error & $\pm 2.3 \%$ & $\pm 32 \%$ \\
\hline \hline
\end{tabular}

$0.60 \pm 0.19$ (stat) ${ }_{-0.58}^{+0.56}$ (syst), respectively. When we unblinded 200 live-days of data, we observed 3 events which were deemed compatible with background. With a predicted background of $0.60 \pm 0.19$ (stat) ${ }_{-0.58}^{+0.56}$ (syst) events, the probabilities of observing one, two or three events due solely to fluctuations in the background are $30 \%, 13 \%$ and $5 \%$, respectively.

We combined the systematic errors in quadrature with the statistical errors and applied a profile log-likelihood method [38] to obtain the confidence interval [39]. The

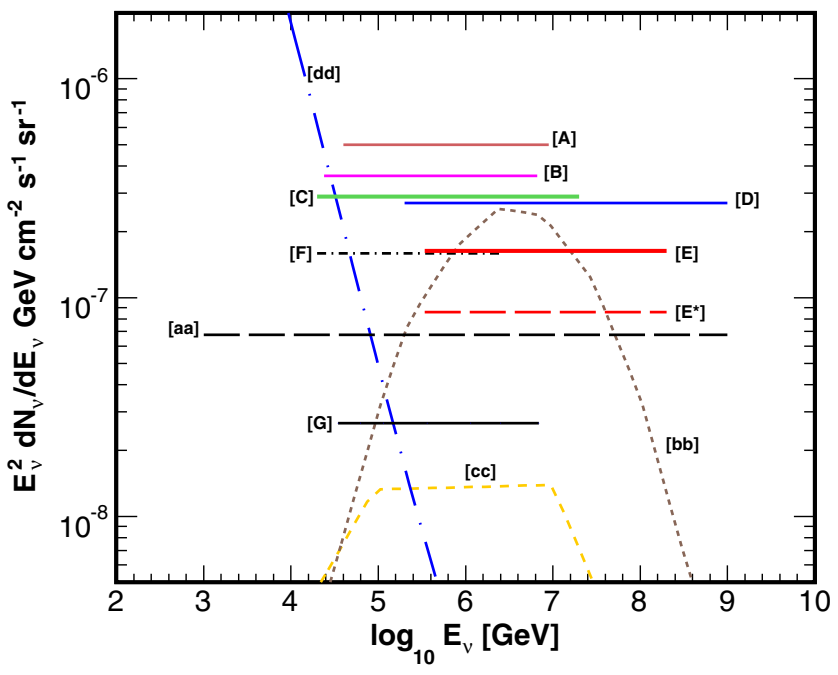

FIG. 18 (color online). The limits on production of UHE neutrinos. [A]: AMANDA-II cascade all-flavor limit (1001 live-days) [40], [B]: IC22 cascade all-flavor limit (257 livedays) [34], [C]: Baikal all-flavor limit (1038 live-days) [42], [D]: AMANDA-II UHE all-flavor limit (457 live-days) [41], [E]: IC22 UHE all-flavor limit from diffuse sources using the analysis described in this paper (this work, 200 live-days), [E*]: IC22 UHE all-flavor sensitivity (this work, 200 live-days), [F]: ANTARES '07-'09 $\nu_{\mu} \times 3$ (334 live-days) [43], [G]: IC40 $\nu_{\mu} \times$ 3 (375.5 live-days) [44], [aa]: Waxman-Bahcall $\left(\nu_{\mu}\right.$ and $\left.\bar{\nu}_{\mu}\right)$ model $1998 \times \frac{3}{2}$ [4], [bb]: Stecker AGN (Seyfert) 2005 [45], [cc]: Waxman-Bahcall Prompt GRB model [4], and [dd]: Atmospheric neutrino flux (Bartol + Sarcevic standard model).
90\% C.L. upper limit on signal for 200 live-days was obtained as $\mu_{90}^{s}=7.7$ events. The $90 \%$ C.L. upper limit on astrophysical all-flavor neutrino flux, $\Phi_{90}\left(\nu_{\mathrm{x}}\right)$, was obtained using the following relation: $\frac{\Phi_{90}}{\Phi_{\mathrm{WB}}}=\frac{\mu_{90}^{s}}{N_{\mathrm{WB}}}$ where $\Phi_{\mathrm{WB}}$ and $N_{\mathrm{WB}}$ are the WB bound for all-flavor astrophysical neutrinos and the corresponding number of all-flavor astrophysical neutrinos for 200 live-days, respectively. The obtained $90 \%$ C.L. upper limit is $E_{\nu}^{2} \Phi_{90}\left(\nu_{\mathrm{x}}\right)<16.3 \times$ $10^{-8} \mathrm{GeV} \mathrm{cm}^{-2} \mathrm{sr}^{-1} \mathrm{~s}^{-1}$ for the 3 observed events from the 200 live-days of IC22 data.

This limit applies to the primary neutrino energy range of $340 \mathrm{TeV}<E_{\nu}<200 \mathrm{PeV}$, covering the middle $90 \%$ of the accepted simulated signal. Figure 18 shows this limit together with several theoretical model predictions. The upper limit on the tau neutrino flux is one-third that of the all-flavor astrophysical neutrino flux if one assumes a flavor ratio of $\nu_{\mathrm{e}}: \nu_{\mu}: \nu_{\tau}=1: 1: 1$ at Earth.

\section{CONCLUSIONS AND OUTLOOK}

A set of selection criteria designed for UHE $\nu_{\tau}$ detection were applied to IceCube data. These criteria also had appreciable efficiency for UHE $\nu_{\mathrm{e}}$ and $\nu_{\mu}$ detection. We applied these criteria to 200 live-days of data from IceCube's 22-string configuration and observed 3 events in the final sample. We therefore set a $90 \%$ C.L. upper limit on the astrophysical UHE all-flavor neutrino flux of $E_{\nu}^{2} \Phi_{90}\left(\nu_{\mathrm{x}}\right)<16.3 \times 10^{-8} \mathrm{GeV} \mathrm{cm}^{-2} \mathrm{sr}^{-1} \mathrm{~s}^{-1}$. The analysis improves on the previous limit set by AMANDA $[34,40,41]$ with comparable integrated exposure. Future IceCube searches specialized for $\nu_{\tau}$ will be more sensitive due to the increased instrumented volume relative to IC22. The large volume will also warrant the application of sophisticated $\nu_{\tau}$ reconstructions, further improving the sensitivity of these searches.

\section{ACKNOWLEDGMENTS}

We acknowledge the support from the following agencies: U.S. National Science Foundation-Office of Polar Programs, U.S. National Science Foundation-Physics Division, University of Wisconsin Alumni Research Foundation, the Grid Laboratory Of Wisconsin (GLOW) grid infrastructure at the University of WisconsinMadison, the Open Science Grid (OSG) grid infrastructure; U.S. Department of Energy, and National Energy Research Scientific Computing Center, the Louisiana Optical Network Initiative (LONI) grid computing resources; National Science and Engineering Research Council of Canada; Swedish Research Council, Swedish Polar Research Secretariat, Swedish National Infrastructure for Computing (SNIC), and Knut and Alice Wallenberg Foundation, Sweden; German Ministry for Education and Research (BMBF), Deutsche Forschungsgemeinschaft (DFG), Research Department of Plasmas with Complex Interactions (Bochum), Germany; Fund for Scientific 
Research (FNRS-FWO), FWO Odysseus programme, Flanders Institute to encourage scientific and technological research in industry (IWT), Belgian Federal Science Policy Office (Belspo); University of Oxford, United Kingdom;
Marsden Fund, New Zealand; Australian Research Council; Japan Society for Promotion of Science (JSPS); the Swiss National Science Foundation (SNSF), Switzerland.
[1] F. Halzen and D. Hooper, Rep. Prog. Phys. 65, 1025 (2002).

[2] J. Becker, Phys. Rep. 458, 173 (2008).

[3] F. W. Stecker, C. Done, M. H. Salamon, and P. Sommers, Phys. Rev. Lett. 66, 2697 (1991).

[4] E. Waxman and J. Bahcall, Phys. Rev. Lett. 78, 2292 (1997); Phys. Rev. D 59, 023002 (1998).

[5] A. Levinson and E. Waxman, Phys. Rev. Lett. 87, 171101 (2001).

[6] J. G. Learned and S. Pakvasa, Astropart. Phys. 3, 267 (1995).

[7] J.P. Rachen and P. Meszaros, Phys. Rev. D 58, 123005 (1998).

[8] T. Kashti and E. Waxman, Phys. Rev. Lett. 95, 181101 (2005).

[9] M. Kachelriess, S. Ostapchenko, and R. Tomas, Phys. Rev. D 77, 023007 (2008).

[10] A. Achterberg et al., Phys. Rev. D 76, 042008 (2007); M. Ackermann et al., Astrophys. J. 675, 1014 (2008).

[11] J. Ahrens et al., Phys. Rev. D 67, 12003 (2003); M. Ackermann et al., Astropart. Phys. 22, 127 (2004).

[12] R. Abbasi et al., Astropart. Phys. 34, 420 (2011).

[13] K. Nakamura et al. (Particle Data Group), J. Phys. G 37, 075021 (2010).

[14] G. D. Barr, P. Lipari, S. Robbins, T. K. Gaisser, and T. Stanev, Phys. Rev. D 70, 023006 (2004); G. D. Barr, T. K. Gaisser, S. Robbins, and T. Stanev, Phys. Rev. D 74, 094009 (2006).

[15] G. Florentini, V. A. Naumov, and F. L. Villante, Phys. Lett. B 510, 173 (2001).

[16] R. Enberg, M. H. Reno, and I. Sarcevic, Phys. Rev. D 78, 043005 (2008).

[17] A. D. Martin, M. G. Ryskin, and A. M. Stasto, Acta Phys. Pol. B 34, 3273 (2003).

[18] F. Halzen and D. Saltzberg, Phys. Rev. Lett. 81, 4305 (1998).

[19] J. G. Learned and S. Pakvasa, Astropart. Phys. 3 , 267 (1995).

[20] R. Abbasi et al., Nucl. Instrum. Methods Phys. Res., Sect. A 618, 139 (2010).

[21] A. Achterberg et al., Astropart. Phys. 26, 155 (2006).
[22] R. Abbasi et al., Nucl. Instrum. Methods Phys. Res., Sect. A 601, 294 (2009).

[23] F. Halzen and S. R. Klein, Rev. Sci. Instrum. 81, 081101 (2010).

[24] A. Gazizov and M. Kowalski, Comput. Phys. Commun. 172, 203 (2005).

[25] E. Bugaev, T. Montaruli, Y. Shlepin, and I. Sokalski, Astropart. Phys. 21, 491 (2004).

[26] S. Iyer, M.H. Reno, and I. Sarcevic, Phys. Rev. D 61, 053003 (2000); 62, 123001 (2000); 66, 077302 (2002).

[27] F. Becattini and S. Bottai, Astropart. Phys. 15, 323 (2001); S. Bottai and S. Giurgola, Astropart. Phys. 18, 539 (2003).

[28] D. Heck, J. Knapp, J. N. Capdevielle, G. Schatz, and T. Thouw, Technischer Report No. 6019, Forschungszentrum Karlsruhe, Germany, 1998, http://www-ik.fzk.de/corsika.

[29] D. Chirkin and W. Rhode, arXiv:hep-ph/0407075v2.

[30] R. Glasstetter et al., in 26th International Cosmic Ray Conference, Salt Lake City, Utah, 1999), edited by D. Kieda, M. Salamon, and B. Dingus (AIP, New York, 2001.

[31] J. R. Hörandel, Astropart. Phys. 19, 193 (2003).

[32] J. Ahrens et al., Nucl. Instrum. Methods Phys. Res., Sect. A 524, 169 (2004).

[33] G. C. Hill and K. Rallins, Astropart. Phys. 19, 393 (2003).

[34] R. Abbasi et al., Phys. Rev. D 84, 072001 (2011).

[35] M. Ackermann et al., J. Geophys. Res. 111, D13203 (2006).

[36] D. Chirkin, for the IceCube Collaboration, Proceedings Study of South Pole ice transparency with IceCube flashers, 32nd ICRC, Beijing, China, 2011.

[37] M. M. Block, P. Ha, and D. W. McKay, Phys. Rev. D 82, 077302 (2010).

[38] J. Lundberg, J. Conrad, W. Rolke, and A. Lopez, Comput. Phys. Commun. 181, 683 (2010).

[39] G. J. Feldman and R. D. Cousins, Phys. Rev. D 57, 3873 (1998).

[40] R. Abbasi et al., Astropart. Phys. 34, 420 (2011).

[41] M. Ackermann et al., Astrophys. J. 675, 1014 (2008).

[42] A. V. Avrorin et al., Astron. Lett. 35, 651 (2009).

[43] J. A. Aguilar et al., Phys. Lett. B 696, 16 (2011).

[44] R. Abbasi et al., Phys. Rev. D 84, 082001 (2011).

[45] F. W. Stecker, Phys. Rev. D 72, 107301 (2005). 